\title{
Wearable materials with embedded synthetic biology sensors for biomolecule detection
}

\author{
Peter Q. Nguyen $\mathbb{1}^{1,2,10}$, Luis R. Soenksen ${ }^{1,3,4,10}$, Nina M. Donghia', Nicolaas M. Angenent-Mari ${ }^{1,4,5}$, \\ Helena de Puig, ${ }^{1,4}$, Ally Huang 1,4,5, Rose Lee ${ }^{1}$, Shimyn Slomovic ${ }^{1}$, Tommaso Galbersanini ${ }^{6}$, \\ Geoffrey Lansberry ${ }^{1}$ ', Hani M. Sallum ${ }^{1}{ }^{1}$, Evan M. Zhao', James B. Niemi' and
}

James J. Collins $101,4,5,7,8,9 \bowtie$

Integrating synthetic biology into wearables could expand opportunities for noninvasive monitoring of physiological status, disease states and exposure to pathogens or toxins. However, the operation of synthetic circuits generally requires the presence of living, engineered bacteria, which has limited their application in wearables. Here we report lightweight, flexible substrates and textiles functionalized with freeze-dried, cell-free synthetic circuits, including CRISPR-based tools, that detect metabolites, chemicals and pathogen nucleic acid signatures. The wearable devices are activated upon rehydration from aqueous exposure events and report the presence of specific molecular targets by colorimetric changes or via an optical fiber network that detects fluorescent and luminescent outputs. The detection limits for nucleic acids rival current laboratory methods such as quantitative PCR. We demonstrate the development of a face mask with a lyophilized CRISPR sensor for wearable, noninvasive detection of SARS-CoV-2 at room temperature within $90 \mathrm{~min}$, requiring no user intervention other than the press of a button.

S ynthetic biology has enabled unprecedented control of biological systems and has provided a rich palette of modular biosensors, genetic logic gates and output effectors for the design of custom biological circuits ${ }^{1}$. In parallel, recent developments in wireless technology, wearable electronics, smart materials and functional fibers with new mechanical, electrical and optical properties have led to sophisticated biosensing systems ${ }^{2}$. Although genetically encoded sensors have been readily incorporated into bench-top diagnostics, examples of wearable devices using these tools are limited. Only a few demonstrations of hygroscopically actuated vents and response to induction molecules have been achieved using living engineered bacteria encapsulated in flexible substrates and hydrogels in a wearable format ${ }^{3-6}$. This approach encounters several limitations, particularly that of sustaining living organisms in the devices for extended periods. Retaining the viability and function of wearable sensing systems based on living cells requires nutrient delivery and waste extraction, as well as temperature and gas regulation, all of which involve numerous technological hurdles. Genetically engineered cells can also pose biocontainment or biohazard concerns, particularly if integrated into consumer-level garments. Moreover, the mutational pressures on evolving cell populations can result in loss of the genetic phenotype and function. An approach that could resolve the mismatch between the practical requirements of wearable use and the operational limitations of available biomolecular circuits for sensing and response would broaden the applications of wearable materials and may enable assessment of molecular targets difficult to detect through other technologies?

Cell-free synthetic biology reactions are self-contained abiotic chemical systems with all the biomolecular components required for efficient transcription and translation. Such systems can be freeze-dried into shelf-stable formats utilizing porous substrates, which allow for robust distribution, storage and use without specialized environmental or biocontainment requirements ${ }^{8}$. Genetically engineered circuits, encoded in DNA or RNA, can be added to freeze-dried, cell-free (FDCF) reactions for activation by simple rehydration. Robust FDCF systems have already been developed for inexpensive paper-based nucleic acid diagnostics; sensitive programmable CRISPR-based nucleic acid sensors ${ }^{9,10}$; on-demand production of antimicrobials, antibodies and enzymes ${ }^{11}$; and low-cost educational kits for teaching ${ }^{12-14}$. Here we propose the use of FDCF genetic circuits in combination with specifically designed flexible and textile substrates to create practical wearable biosensors. We report on the design and validation of various wearable FDCF (wFDCF) sensors for small molecule, nucleic acid and toxin detection. The sensors are integrated into flexible multi-material substrates (for example, silicone elastomers and textiles) using genetically engineered components, including toehold switches, transcriptional factors, riboswitches, fluorescent aptamers and CRISPR-Cas12a complexes (Supplementary Fig. 1).

\section{Results}

Colorimetric wFDCF wearables. For our first wFDCF demonstration, we embedded colorimetric genetic circuits into cellulose substrates surrounded by a fluid wicking and containment assembly made of flexible elastomers. These prototypes were assembled layer-by-layer to form reaction chambers fluidically connected to top sample portals (Fig. 1a). The devices are flexible, elastic and can rapidly wick in splashed fluids through capillary action

\footnotetext{
'Wyss Institute for Biologically Inspired Engineering, Harvard University, Boston, MA, USA. ${ }^{2}$ School of Engineering and Applied Sciences, Harvard University, Cambridge, MA, USA. ${ }^{3}$ Department of Mechanical Engineering, Massachusetts Institute of Technology, Cambridge, MA, USA. ${ }^{4}$ Institute for Medical Engineering and Science, Massachusetts Institute of Technology, Cambridge, MA, USA. ${ }^{5}$ Department of Biological Engineering, Massachusetts Institute of Technology, Cambridge, MA, USA. ${ }^{6}$ DREAMLUX, Samsara S.r.l., Milan, Italy. ${ }^{7}$ Synthetic Biology Center, Massachusetts Institute of Technology, Cambridge, MA, USA. ${ }^{8}$ Harvard-MIT Program in Health Sciences and Technology, Cambridge, MA, USA. ${ }^{9}$ Broad Institute of MIT and Harvard, Cambridge, MA, USA. ${ }^{10}$ These authors contributed equally: Peter Q. Nguyen, Luis R. Soenksen.凶e-mail: jimjc@mit.edu
} 


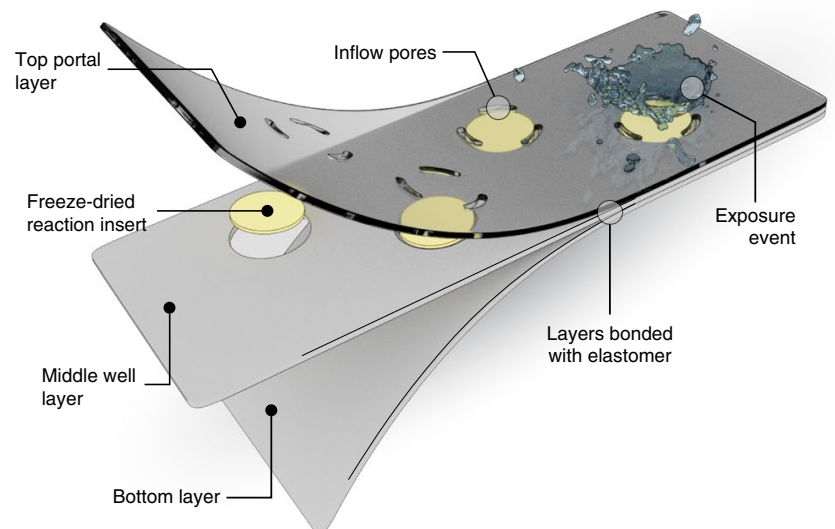

b

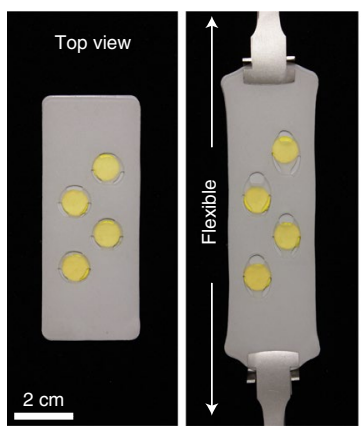

c

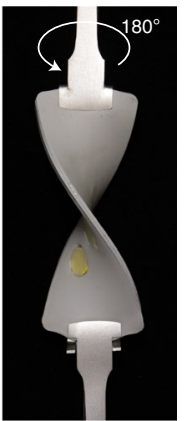

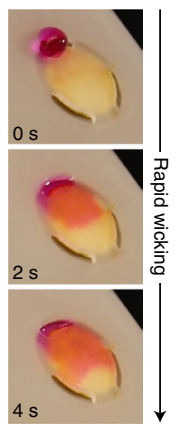

f
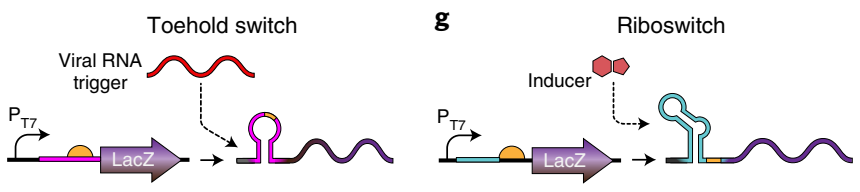
0000 Inducer (aTc)
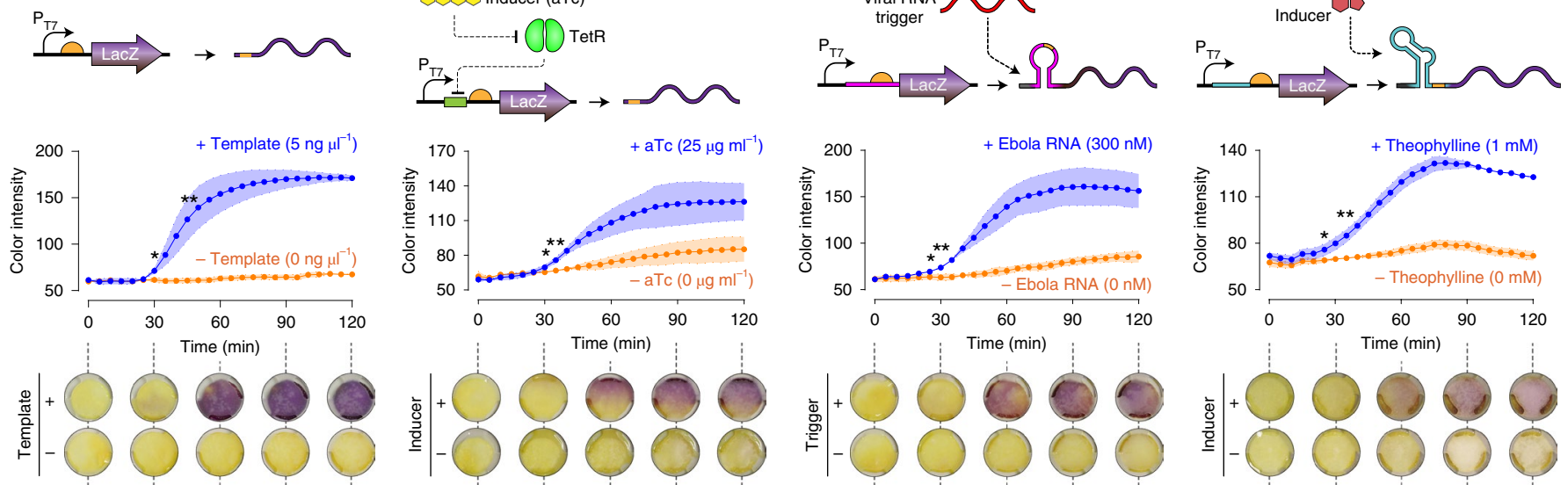

Fig. 1 | Wearable cell-free synthetic biology. a, Schematic of the layer-by-layer assembly of the wearable devices. Each layer is fabricated from skin-safe silicone elastomer. The FDCF reactions are embedded in a cellulose matrix placed within each chamber. $\mathbf{b}$, An array of assembled reaction chambers showing the elasticity (center) and flexibility (right) of the devices. c, Portals cut into the outermost layer allow sample access, which is rapidly drawn into the reaction chambers through capillary action. The hydrophobic chamber walls prevent inhibitory dilution through lateral diffusion. $\mathbf{d}-\mathbf{g}$, Various types of synthetic biology circuits can be freeze-dried in these wearable devices, for example, constitutively expressed outputs such as Lac $Z$ ( $P=0.015$ at $30 \mathrm{~min},{ }^{\star \star} P=0.008$ at $45 \mathrm{~min}$ ) (d); transcription-factor-regulated circuits for small-molecule detection, here shown regulated by Tet $R$ ( ${ }^{\star} P=0.03$ at $30 \mathrm{~min},{ }^{\star \star} P=0.003$ at $35 \mathrm{~min}$ ) (e); toehold switches for nucleic acid sensing such as the demonstrated Ebola RNA-targeting toehold ( ${ }^{\star} P=0.04$ at $25 \mathrm{~min},{ }^{\star \star} P=0.007$ at $\left.30 \mathrm{~min}\right)(\mathbf{f})$; and riboswitches to detect various small molecules such as the theophylline-activated riboswitch ( ${ }^{\star} P=0.05$ at $25 \mathrm{~min}$, ${ }^{\star \star} P=0.005$ at $\left.35 \mathrm{~min}\right)(\mathbf{g})$. Each plot shows mean (line) \pm s.d. (shaded region) of integrated green channel values from color-deconvoluted images from $n=3$ independently fabricated and tested wFDCF reaction chambers. Statistical significance between the two groups as indicated at specific time points was compared using an unpaired two-tailed Student's $t$-test. Bottom images are representative color images of the wearable device. aTc, anhydrotetracycline.

(Fig. 1b,c). Pinning geometries throughout the device direct sample fluids towards enclosed hydrophilic paper networks allowing for reaction rehydration (Fig. 1c and Supplementary Fig. 3b). Using an lac $Z \beta$-galactosidase operon as the circuit output to hydrolyze chlorophenol red- $\beta$-D-galactopyranoside (CPRG), a yellow-to-purple color change develops upon exposure to a target (Supplementary Figs. $2 \mathrm{~b}$ and $3 \mathrm{a}$ ).

We considered key environmental factors in the design of these prototypes. For instance, sample exposure in the field likely occurs with variable splash volumes (as little as $50-100 \mu \mathrm{l}$ ), relative humidity $(20-40 \%)$ and temperature $\left(20-37^{\circ} \mathrm{C}\right)$. Thus, we optimized our design to reduce inhibition of genetic circuit operation due to evaporation or excessive dilution of components. In particular, our devices use impermeable chambers exhibiting low evaporation rates $(<20 \%$ volume per $\mathrm{h}$ ), which also constrain the rehydration volume to $\sim 50 \mu \mathrm{l}$ per sensor. In addition, the wFDCF reactions were optimized to generate a higher concentrated reaction upon rehydration. We found that $\mathrm{a} \times 1.5$-concentrated cell-free reaction increased the reaction kinetics to enable signal output at least 10 min faster, ensuring that the desired circuit is completed before eventual evaporation in the device terminates the reaction (Extended Data Fig. 2). The resulting stand-alone colorimetric system is modular and can be used in garments such as bracelets (Supplementary Fig. 3c).

We tested this colorimetric wearable platform using four different synthetic biology biosensors with lacZ as the output (Fig. 1d-g). These demonstrations include a constitutive lac $Z$ expression reaction (Fig. 1d), a transcription-factor-regulated circuit using the tetracycline repressor (TetR) for the detection of anhydrotetracycline (Fig. 1e), a toehold switch for the detection of Ebola virus RNA (Fig. 1f) and a theophylline riboswitch for small-molecule sensing (Fig. 1g). The TetR sensor shows that our colorimetric platform can integrate well-established transcription-factor-based modules into a wearable format (Fig. 1e). The toehold sensor enabled detection of Ebola virus RNA at a concentration of $300 \mathrm{nM}$ at $30 \mathrm{~min}$ compared with a control sample containing no target (unpaired $t$-test of independent reaction chambers; deconvoluted green channel 
$P=0.0074$ ) (Fig. 1f). The riboswitch circuit was able to detect its target molecule, theophylline, at a concentration of $1 \mathrm{mM}$ at $35 \mathrm{~min}$ compared with a control sample (unpaired $t$-test of independent reaction chambers; deconvoluted green channel $P=0.005$ ) (Fig. 1g). All of the colorimetric wFDCF sensors reported here exhibited visible changes within $\sim 40-60 \mathrm{~min}$ after exposure to the respective trigger molecules or inducer, and were performed at ambient conditions of $30-40 \%$ relative humidity and $30^{\circ} \mathrm{C}$ to simulate the average skin surface temperature ${ }^{15}$.

Fluorescent wFDCF devices with fiber optic detection for enhanced sensing. Next, we immobilized and activated FDCF sensors in wearable woven fabrics and individual threads. Figure 2 presents various demonstrations of a highly sensitive, textile-based system (Fig. 2a,b) capable of containing and monitoring the activation of wFDCF reactions with fluorescent (Fig. 2c-e, Extended Data Figs. 4-7 and Supplementary Figs. 7 and 14) or luminescent (Fig. $2 \mathrm{f}$ and Extended Data Fig. 3) outputs. To achieve this, we integrated: (1) hydrophilic threads ( $85 \%$ polyester $/ 15 \%$ polyamide) for cell-free reagent immobilization, (2) patterns of skin-safe hydrophobic silicone elastomers for reaction containment, and (3) inter-weaved polymeric optic fibers (POFs) for signal interrogation (Fig. 2a,b, Extended Data Fig. 2 and Supplementary Fig. 8). This fabric was chosen as our main immobilization substrate after conducting a compatibility screening of over 100 fabrics (for example, silks, cotton, rayon, linen, hemp, bamboo, wool, polyester, polyamide, nylon and combination materials) using a lyophilized constitutive lac $Z$ cell-free reaction (Supplementary Figs. 4-6). The analysis of sensor outputs was executed using a custom-built wearable POF spectrometer (Fig. 2b and Extended Data Fig. 8) that could be monitored with a mobile phone application (Supplementary Fig. 11). Using this integrated platform, we performed distributed on-body sensing of various target exposures as shown in Fig. $2 \mathrm{c}-\mathrm{f}$. A sample activation through fluid splashing is shown in Fig. 2a, where the sample wicks through the entry ports with blackout fabrics to rehydrate the FDCF synthetic biology reactions immobilized within the hydrophilic textile fibers. These fibers are located within the excitation and emission layers of the device as shown in Fig. 2a,b. Trigger presence in the splash fluid leads to activation of the sensor circuits, which produce fluorescent or luminescent reporters.

We first verified the function of this textile platform in fluorescence mode using two independent synthetic biology modules upstream of a superfolder green fluorescent protein (sfGFP) operon. These demonstrations included the activation of constitutive sfGFP expression (Fig. 2c) and sensing of theophylline using an inducible riboswitch (Fig. 2d). A third fluorescence demonstration was done via activation of a 49-nucleotide Broccoli aptamer (Fig. 2e) with substrate specificity to (Z)-4-(3,5-difluoro-4-hydroxybenzylidene)2-methyl-1-(2,2,2-trifluoroethyl)-1H-imidazol-5(4H)-one $(\text { DFHBI-1T })^{16}$. Furthermore, demonstrations utilizing luminescence outputs were conducted using a nanoluciferase (nLuc) ${ }^{17}$ operon downstream of a HIV RNA toehold switch (Fig. $2 \mathrm{f}$ and Extended Data Fig. 3a), as well as a Borrelia burgdorferi RNA toehold switch for the wearable detection of Lyme disease (Extended Data Fig. 3b).

Additionally, we tested the operation of our platform for the detection of chemical threats such as organophosphate nerve agents used in chemical warfare and the pesticide industry. To achieve this, we modified our POF platform optics for excitation and detection at near-infrared (NIR) fluorescence, generated from a lyophilized acetylcholinesterase (AChE)-choline oxidase-HRP-coupled enzyme reaction (Fig. 2g). In the presence of acetylcholine, this reaction can produce NIR fluorescence that is readily detectable with our wearable prototype. When exposed to an organophosphate AChE inhibitor, the sensor fluorescence is reduced as compared with unexposed controls. Our wearable nerve agent sensor was validated using paraoxon-ethyl as a nerve agent simulant at levels that are four orders of magnitude lower than the reported lethal dose $\left(\mathrm{LD}_{50}\right)$ by dermal absorption in mammals ${ }^{18}$.

The fluorescent wFDCF platform allows for continuous monitoring of all reaction chambers through the fiber optic network at user-defined sampling intervals for the automated detection of rehydration events and fluorescent outputs from target-activated circuits. This is achieved by illuminating the wFDCF textile reaction with blue light $(447 \mathrm{~nm})$ via etched excitation POFs (Fig. 2b and Extended Data Fig. 2). The light emitted from the activated system is then collected by the second set of emission POFs, which exit the fabric weave and bundle into a single trunk connected to the optical sensor of our wearable spectrometer (Extended Data Figs. 2 and 8). Signals coming from each of the devices are filtered and processed to generate temporally and spatially resolved fluorescence images of the POF bundle-ends $(510 \mathrm{~nm})$ and averaged pixel intensity traces per channel for quantitative analysis (Fig. 2b). In the case of luminescence demonstrations, all POF bundles are treated as signal emission sensors, without the need for sample illumination. All reported wFDCF fluorescence and luminescence sensor replicates $(n \geq 3)$ exhibited visible fluorescence or luminescence within 5-20 min after exposure to relevant trigger conditions, at 30-40\% relative humidity and $30^{\circ} \mathrm{C}$.

CRISPR-based wFDCF sensors enable direct nucleic acid detection in wearables. Sensors based on programmable CRISPR and CRISPR-associated (Cas) enzymes ${ }^{9,19,20}$ have several advantages over other biosensors, including high sensitivity, rapid output, single

Fig. 2 | Design and validation of fluorescent and luminescent FDCF synthetic biology wearables. a, Fiber optic-embedded textiles allow excitation and emission detection of rehydrated lyophilized biosensors. Bottom, an example rehydration event shows the aqueous sample being wicked through the portals into internal reaction wells. b. Top, side diagram showing the layers of the assembled device. Contaminated splashes access the device interior through portals in the top layer. Bottom, interior view of the device, where two layers of hydrophobically patterned fabric inter-woven with POFs in a coplanar arrangement allows for embedding of FDCF reaction components and excitation/emission lighting. Excitation POFs are illuminated by LED arrays and emission POFs are bundled to an optical sensor containing a filter (for fluorescence only) and collimating lens. c, Fluorescent signal after rehydration of wFDCF constitutive sfGFP template as compared with control. Fluorescence is statistically distinguishable from the control after 14 min $\left({ }^{\star} P=0.04\right)$ and $20 \mathrm{~min}\left({ }^{\star \star} P=0.002\right)$. d, Activation of wFDCF riboswitch with $1 \mathrm{mM}$ theophylline compared with $0 \mathrm{mM}$ theophylline control. Statistically distinguishable signals after $19.5 \mathrm{~min}\left({ }^{\star} P=0.04\right)$ and $25 \mathrm{~min}\left({ }^{\star}{ }^{*} P=0.008\right)$. e, Wearable demonstration of fluorescent aptamer being activated by the presence of $50 \mu \mathrm{M}$ DFHBI-1T substrate compared with no substrate control. Fluorescent signal is statistically distinguishable after $24.5 \mathrm{~min}\left({ }^{\star} P=0.04\right)$ and $35 \mathrm{~min}$ $\left.{ }^{\star \star} P=0.01\right) . \mathbf{f}$, An HIV toehold sensor with luminescence output. HIV RNA trigger was added at $10 \mu \mathrm{M}$ and was statistically distinguishable from the control ( ${ }^{\star} P=0.01$ at $6 \mathrm{~min},{ }^{\star \star} P=0.005$ at $\left.7 \mathrm{~min}\right) . \mathbf{g}$, Wearable detection of organophosphate nerve agents using a lyophilized HRP-coupled enzyme sensor with $50 \mathrm{mM}$ acetylcholine with and without $3.7 \mathrm{mg} \mathrm{ml}^{-1}$ paraoxon-ethyl (AChE inhibitor). Statistical differences from controls are indicated $\left({ }^{\star} P=0.03\right.$ at $7 \mathrm{~min},{ }^{\star \star} P=0.001$ at $9 \mathrm{~min}$ ). All images above plots are recorded POF bundle images synchronized with the reaction time profiles. All plots show mean pixel intensity values (dark data points) \pm s.d. (light colored region). Each experiment is a total of $n=9$ fiber optic outputs per condition. Any fibers that were $1 \mathrm{~s}$.d. below the mean of all nine fiber outputs were excluded. Statistical significance was determined by unpaired one-tailed Student's $t$-test. Scale bars in brightfield images are $250 \mu \mathrm{m}$. ChOx, choline oxidase; Em, emission; Ex, excitation; RFU, relative fluorescence units. 
base-pair resolution, freeze-drying compatibility and programmability to target any DNA or RNA sequence through interchangeable guide RNAs (gRNAs). Thus, we integrated CRISPR-based specific high-sensitivity enzymatic reporter unlocking (SHERLOCK) sensors into our fluorescence wFDCD platform to demonstrate this detection technique in wearable applications (Fig. 3a). We

a
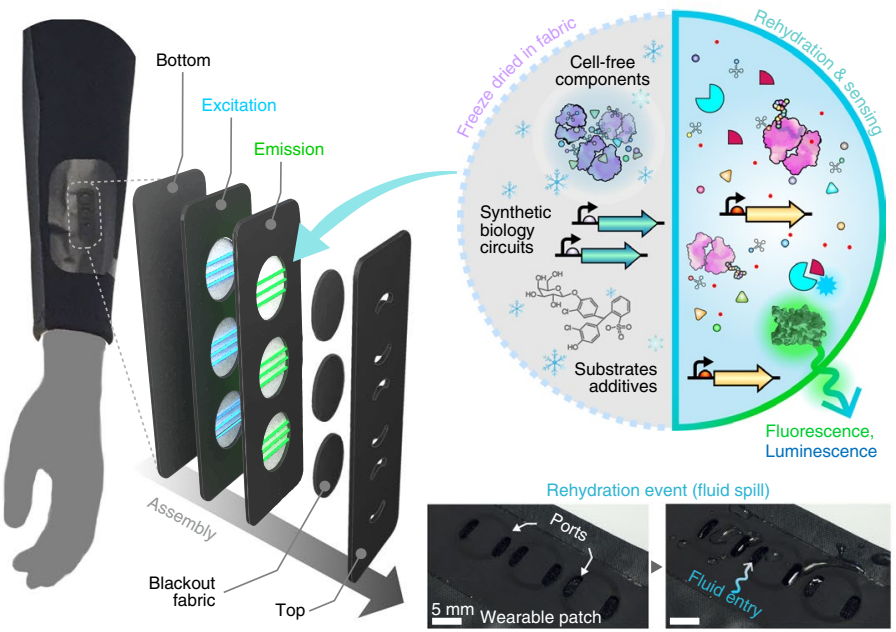

b
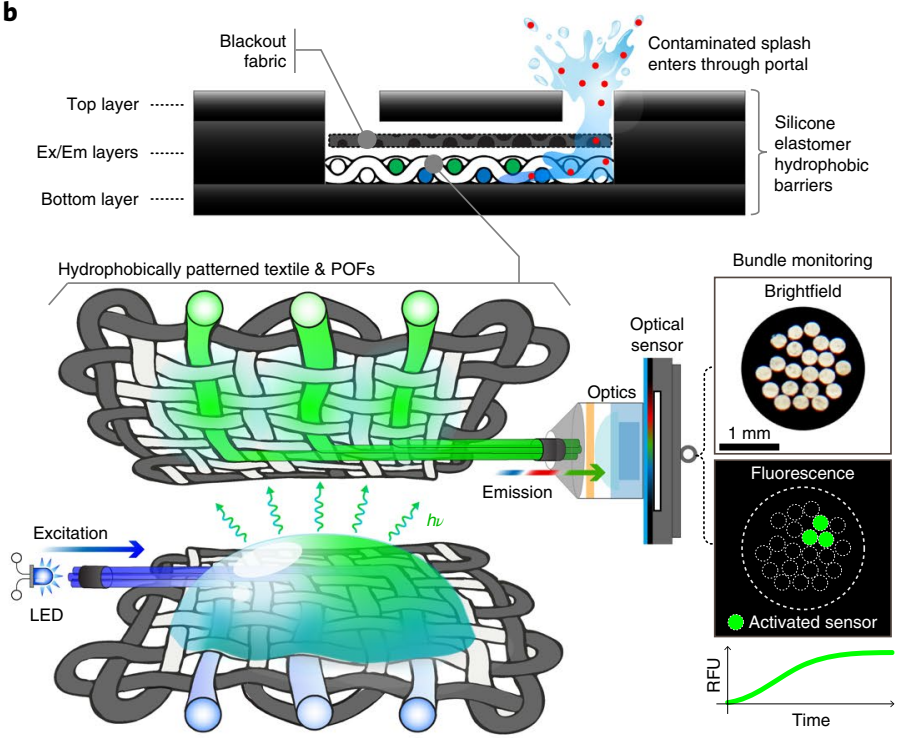

c

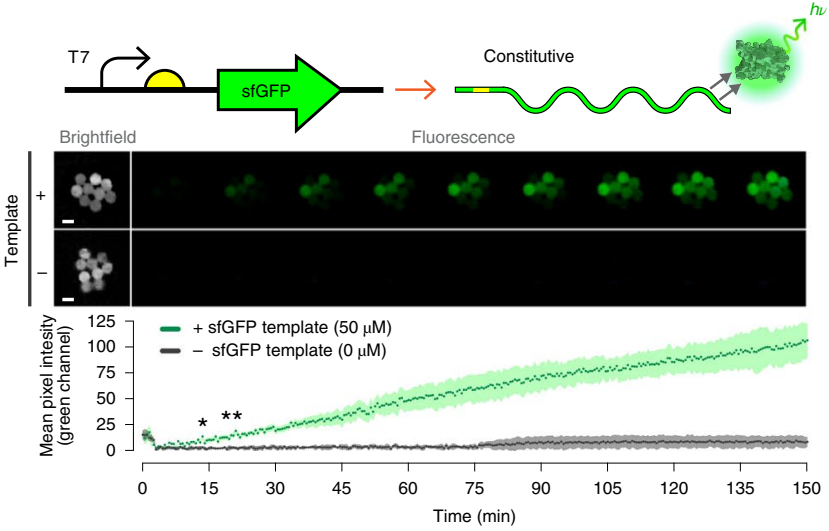

used Cas13a and Cas12a for the detection of RNA and DNA, respectively. For DNA detection, we used a Cas12a ortholog from Lachnospiraceae bacterium (LbaCas12a) ${ }^{19,21}$ that displays a nonspecific collateral cleavage activity towards single-stranded DNA (ssDNA) after detection of a gRNA-defined double-stranded DNA (dsDNA) target. This Cas12a-based sensor was paired with

d

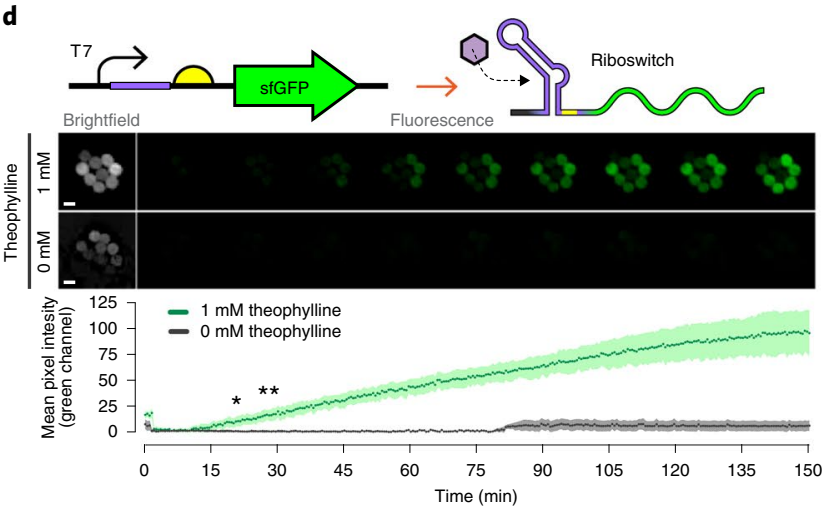

e
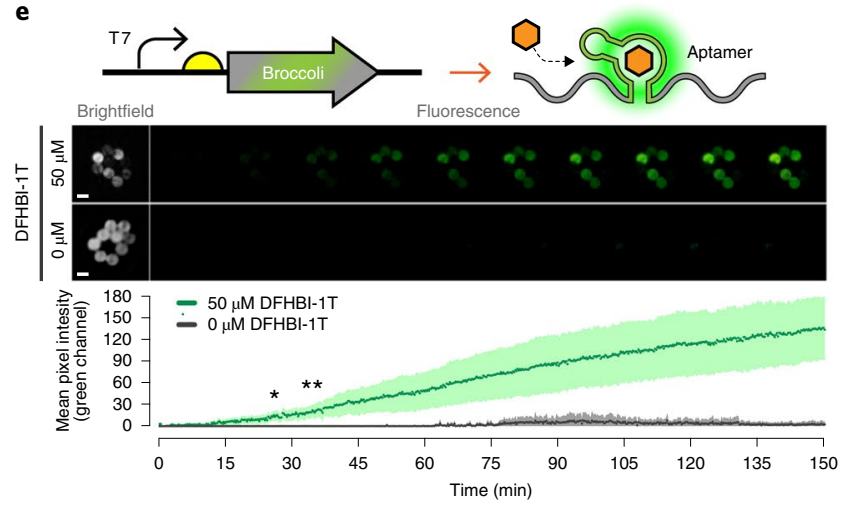

$\mathbf{f}$
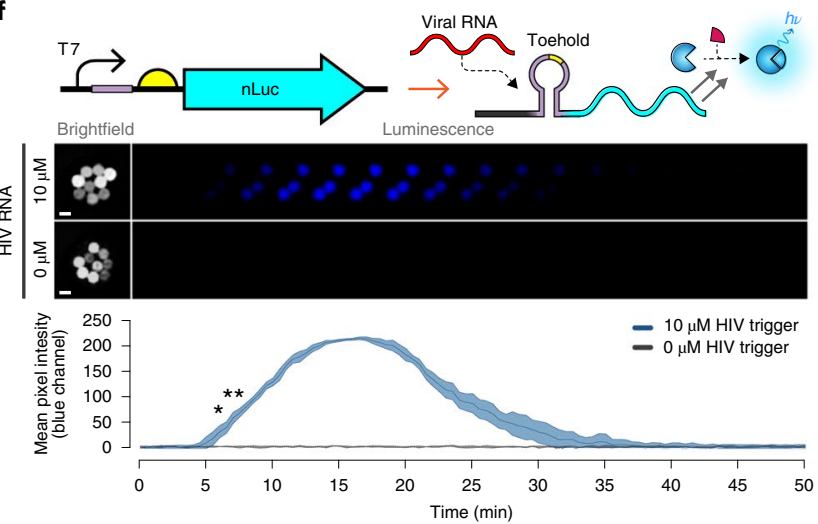

g Organophosphate nerve agent
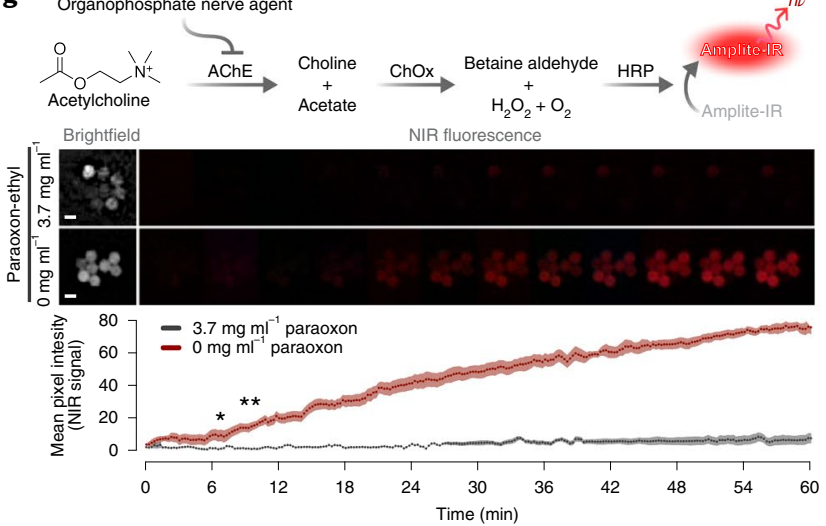


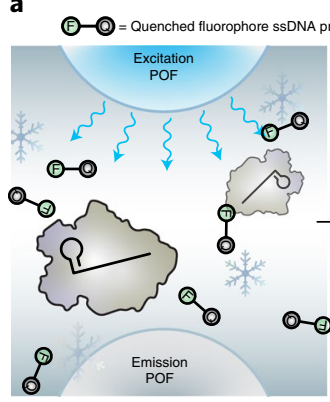

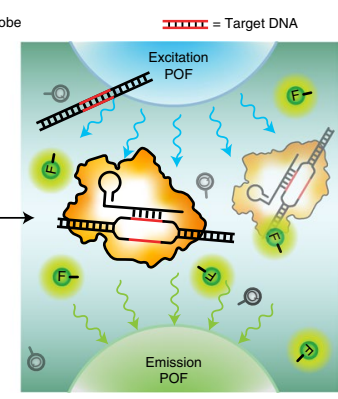

G Normalized signal intensity $(60 \mathrm{~min})$

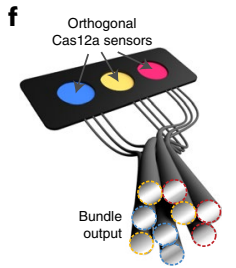

i
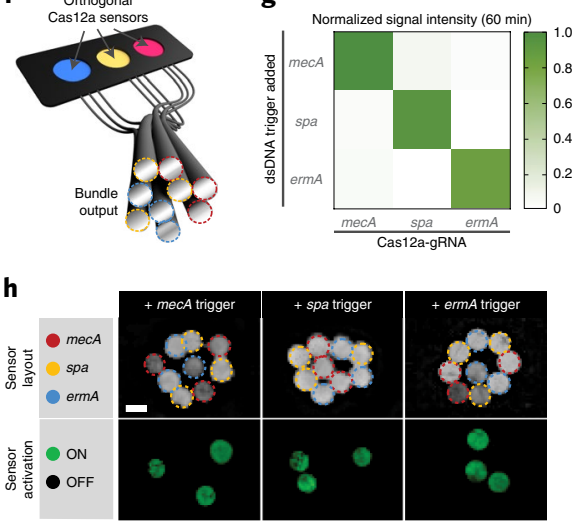

b

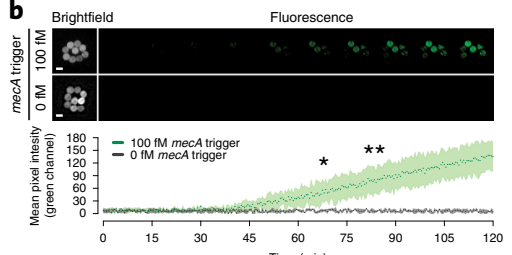

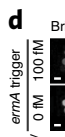
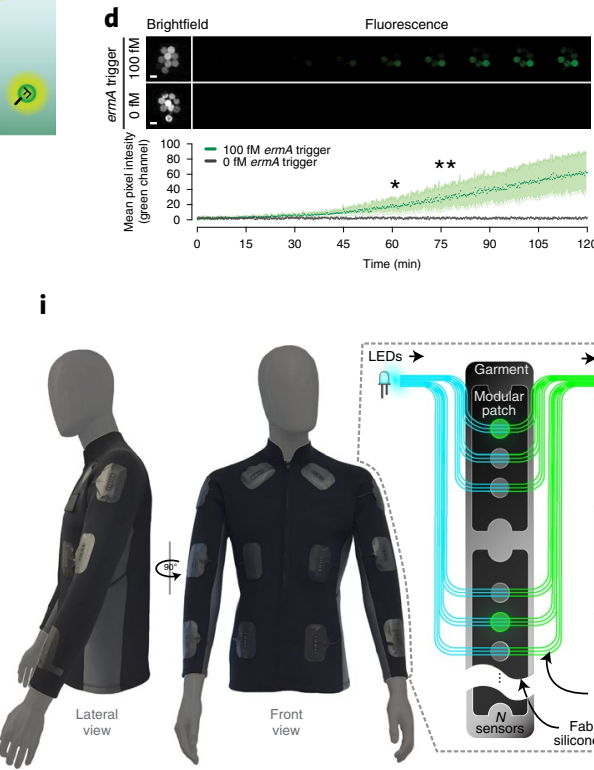
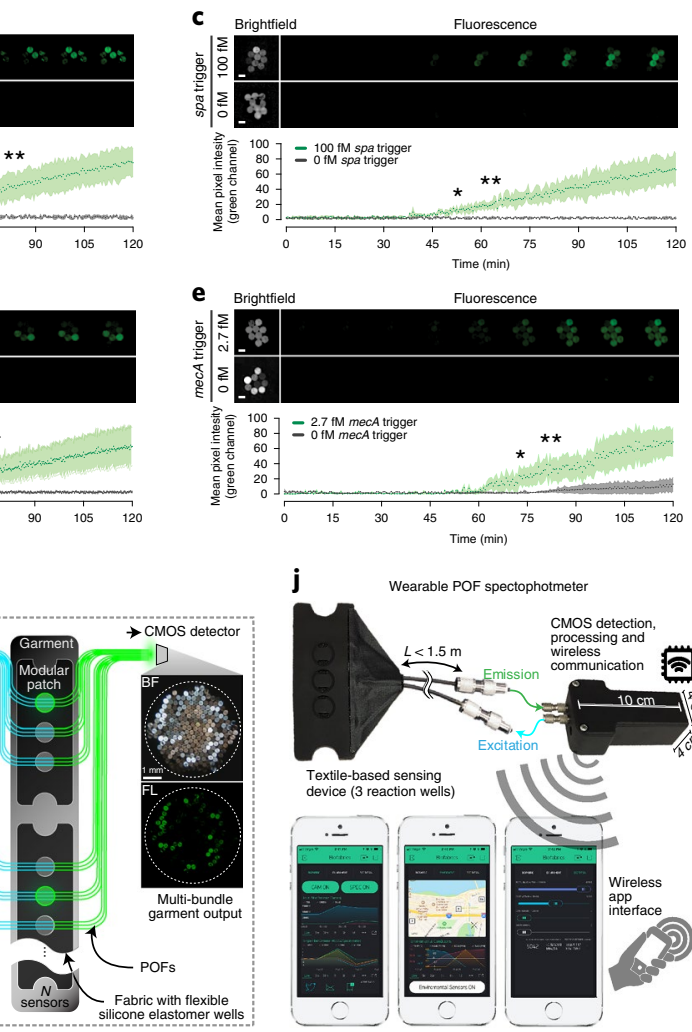

j

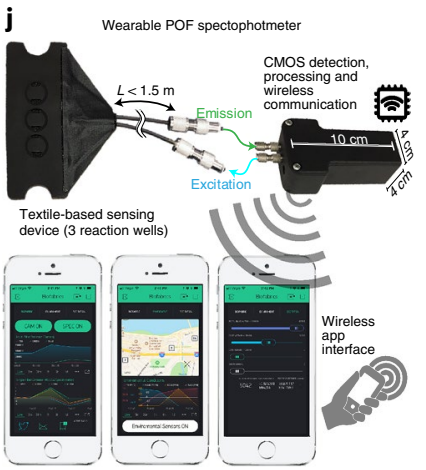

Fig. 3 | Validation of CRISPR-based FDCF wearable sensors. a, The sensing mechanism of the CRISPR-Cas12a system is based on catalytic trans-cleavage of fluorophore-quencher ssDNA probes after activation by an RPA-amplified dsDNA trigger. $\mathbf{b}$, wFDCF mecA CRISPR-based sensor exposed to sample containing $100 \mathrm{fM}$ mecA trigger. Statistically distinguishable signals are indicated for specific time points $\left({ }^{\star} P=0.04\right.$ at $68 \mathrm{~min},{ }^{\star \star} P=0.007$ at $\left.76 \mathrm{~min}\right)$. c, wFDCF spa CRISPR-based sensor exposed to $100 \mathrm{fM}$ spa trigger ( ${ }^{\star} P=0.03$ at $51 \mathrm{~min}$, ${ }^{\star \star} P=0.006$ at $\left.62 \mathrm{~min}\right)$. d, wFDCF ermA CRISPR-based sensor exposed to $100 \mathrm{fM}$ ermA trigger ( ${ }^{\star} P=0.04$ at $20 \mathrm{~min},{ }^{\star \star} P=0.009$ at $\left.60 \mathrm{~min}\right)$. e, Experimental detection of mecA CRISPR-based sensor at $2.7 \mathrm{fM}$ trigger was statistically distinguishable ( ${ }^{\star} P=0.04$ at $72 \mathrm{~min},{ }^{\star \star} P=0.001$ at $82 \mathrm{~min}$ ), corresponding to 10,000 dsDNA-copies per $\mu$. Plots b-e show mean pixel intensity (dark data points) \pm s.d. (light colored region) from three independent wells, each having three fiber optic sensors, for a total of $n=9$ fiber optic outputs. Any fibers below 1s.d. of the mean of all nine fiber outputs were excluded from analysis. Statistical significance calculated as unpaired one-tailed Student's $t$-test is indicated for specific time points. f, Orthogonality demonstration of mecA/spa/ermA CRISPR-based multi-sensor wearables. g, Rehydration only yielded activation of sensors when the Cas12a-gRNA sensor was in the presence of its programmed trigger dsDNA as shown by comparing signal intensities from all sensor-trigger combinations. h, Example fluorescence activation of different CRISPR-based sensors upon exposure to specific dsDNA triggers. These experiments were repeated three times with similar results. Scale bars shown are $250 \mu \mathrm{m}$. i, Garment-level integration of fabric-based wearable synthetic biology sensors. Distributed continuous sensing of garment activity can be achieved through multi-bundle imaging. j, Connection of fabric-based module to wearable POF spectrometer with wireless connectivity capabilities. The spectrometer electronics consist of a Raspberry Pi Zero W with a camera module (Raspberry Pi Foundation), as well as LED illumination, environmental sensing and custom-fabricated shields for battery power. The smartphone application for visualization and alarm of wFDCF sensor activation was based on the blynk.io platform (Blynk) which provides support for Raspberry Pi communication. This application allows for wireless recording of experiments and control of device parameters, as well as environmental and geolocation information. CMOS, complementary metal-oxide-semiconductor; L, length.

recombinase polymerase amplification $(\mathrm{RPA})^{22}$ and freeze-dried into a one-pot reaction. In the presence of a target dsDNA sequence, isothermally generated RPA amplicons activate Cas12a-gRNA complexes. Then, active Cas12a engages in trans-ssDNase activity and cleaves quenched ssDNA fluorophore probes, resulting in a fluorescence output (Fig. 3a).

For wearable demonstrations, we designed gRNAs against three common resistance markers in Staphylococcus aureus: the mecA gene common in methicillin-resistant $S$. aureus (MRSA) ${ }^{23}$, the spa gene which encodes the protein A virulence factor ${ }^{24}$ and the ermA gene conferring macrolide resistance ${ }^{25}$. When tested in wFDCF format, the RPA-Cas12a sensors displayed detectable signals within 56-78 min $(P<0.05)$ with femtomolar limits of detection (Fig. 3b-d). Moreover, using our mecA wFDCF sensor (Fig. 3e and Extended Data Fig. 4), we confirmed single-digit femtomolar sensitivity ( $2.7 \mathrm{fM})$. Compatibility with RNA inputs and other CRISPR enzymes such as Cas13a, an ortholog from Leptotrichia wadei bacterium (LwaCas13a) ${ }^{9}$, was also confirmed (Extended Data Fig. 5), exhibiting similar in-device activation dynamics as that of cell-free reactions conducted in a plate reader. These results suggest that our wearable textile platform could be adapted to achieve sensitivities rivaling those of current laboratory diagnostic tests such as quantitative PCR (qPCR) for monitoring contamination or spread of bacteria and viruses.

To further demonstrate the modularity of our CRISPR-Cas12a wearable sensors, we tested wFDCF devices containing three orthogonal Cas12a-gRNA complexes in isolated reaction wells (Fig. 3f). In this experiment, each device was splashed with dd- $\mathrm{H}_{2} \mathrm{O}$ containing different targets, each specific to only one Cas12a-gRNA complex. The orthogonal behavior of our CRISPR-based wearable sensors is shown in Fig. 3g,h, where higher fluorescence was observed for the cases in which the dsDNA trigger matched the predefined Cas12agRNA complex at each sensor location. 
With the goal of real-time monitoring of environmental exposure and biohazard detection, we designed a jacket containing a distributed arrangement of wFDCF multi-sensor arrays (Fig. 3i). The various optical fibers carrying the output emission signals from different sensors can be routed into a single bundle for centralized imaging analysis, which we demonstrate using a wFDCF CRISPR-Cas12a-based MRSA-sensing array, containing spa, ermA and mecA sensors, that was activated in the wearable prototype with a fluid splash containing $100 \mathrm{fM}$ of spa DNA trigger (Extended Data Fig. 6). Only the well containing the spa sensor generated a fluorescent signal upon activation. The platform is also compatible with transcription-only outputs, such as rehydrated fluorescent aptamer reactions (Supplementary Fig. 9), where the fluorescence signal is monitored by microscopy over time.

In addition, the optical sensor allows for facile fluorescent output multiplexing simply by using fluorescent proteins with orthogonal emission profiles (Extended Data Fig. 7). In this example, wFDCF reactions for three constitutively expressed fluorescent output proteins (eforRed ${ }^{26}$, dTomato ${ }^{27}$ and sfGFP ${ }^{28}$ ) were used to detect distinguishable output signals in a single bundle. We also show that the wFDCF POF system is fully compatible with integrated lyophilized lysis components, allowing for the release and detection of a plasmid-borne mecA gene when challenged with intact bacterial cells (Supplementary Fig. 10). Finally, to develop a complete data feedback cycle between the platform and the user, we integrated the detector system with a custom wireless mobile application that enables continuous cloud-based data logging, signal processing, geolocation tracking and on-the-fly control of various detector components through a smartphone or other networked digital device (Fig. 3j). All images and spectral data presented in Figs. 2 and 3 were collected and processed using wFDCF devices fully integrated with our wearable spectrometer and mobile phone application. Further details on the hardware and software design, as well as implementation of an Opuntia microdasys bioinspired fluid collection $^{29}$ add-on for improved sample harvesting and routing splashes outside of the sensor zones into the wFDCF modules, can be found in Extended Data Fig. 8 and Supplementary Figs. 11 and 12.

A face-mask-integrated sensor for SARS-CoV-2 detection in exhaled aerosols. Finally, we explored whether our wFDCF system could be adapted to create face masks capable of detecting SARS-CoV-2, as a complementary approach to diagnosis based on nasopharyngeal sampling. Respiratory droplets and aerosols are the transmission routes for respiratory infectious diseases but have been underutilized historically for diagnosis. Work on breath-based sensing has focused on the detection of volatile organic compound biomarkers in infected patients using electrochemical sensors ${ }^{30,31}$ or downstream mass spectrometry analysis ${ }^{32}$, which may be challenging to implement on a wide scale. The National Institutes of Health Rapid Acceleration of Diagnostics Initiative has identified SARS-CoV-2 detection from breath sampling technologies as an active area of interest for alleviating testing bottlenecks ${ }^{33}$.

The virus accumulates on the inside of masks as a result of coughing, talking or normal respiration ${ }^{34-36}$. We designed a face-mask sensor containing four modular components: a reservoir for hydration, a large surface area collection sample pad, a wax-patterned $\mu$ PAD (microfluidic paper-based analytical device) and a lateral flow assay (LFA) strip (Fig. 4a,b). Each module can be oriented on the outside or inside of the face mask, with the exception of the collection pad, which must be positioned on the mask interior facing the mouth and nose of the patient. Capillary action wicks any collected fluid and viral particles from the sample collection pad to the $\mu \mathrm{PAD}$, which contains an arrangement of freeze-dried lysis and detection components (Fig. 4c). The use of the $\mu \mathrm{PAD}$ format allowed us to rapidly prototype and optimize a passively regulated multi-step reaction process. Each reaction zone is separated by polyvinyl alcohol (PVA) time delays that enable tunable incubation times between each reaction, greatly improving the efficiency of the sensor compared with that of a one-pot lyophilized reaction (Supplementary Fig. 13). The first $\mu \mathrm{PAD}$ reaction zone contains lyophilized lysis reagents including components known to lyse viral membranes ${ }^{37-39}$. The second $\mu \mathrm{PAD}$ reaction zone is a reverse transcription-recombinase polymerase amplification (RT-RPA) reaction zone containing a customized isothermal amplification reaction developed to target a nonoverlapping region of the SARS-CoV-2 S gene. The final $\mu$ PAD reaction zone contains a Cas12a SHERLOCK sensor with an optimized gRNA for detection of the amplified dsDNA amplicon. In the presence of SARS-CoV-2-derived amplicons, the activated Cas12a enables trans-cleavage of a co-lyophilized 6-FAM(TTATTATT)-Biotin ssDNA probe. To enable a simple colorimetric visual readout, an integrated LFA strip is used to detect probe cleavage. The output strip orientation is adjustable to preserve patient confidentiality. Details on the design, performance and relevant molecular sensor sequences are presented in Supplementary Figs. 14 and 15.

From activation of the face-mask sensor to a final readout only takes $\sim 1.5 \mathrm{~h}$. The limit of detection observed for our sensors is 500 copies $(17 \mathrm{aM})$ of SARS-CoV-2 in vitro transcribed (IVT) RNA, which matches that of World Health Organization-endorsed standard laboratory-based RT-PCR assays ${ }^{40}$ (Fig. 4d,e). The sensors also do not cross-react to RNA from other commonly circulating human coronavirus strains (HCoVs) (Fig. 4f,g). Notably, our hands-off diagnostic reaction proceeds to full completion even at room temperature, which is considered suboptimal for RT, RPA and Cas12a activities. We also validated the SARS-CoV-2 face-mask sensor using a precision lung simulator attached to a high-fidelity

Fig. 4 | A face-mask-integrated SARS-CoV-2 wearable diagnostic. a, Schematic of the sensor components. Puncture of the water blister reservoir results in flow through wicking material, moving viral particles collected from the wearer's respiration from the sample collection zone to downstream freeze-dried reactions integrated into a $\mu$ PAD device. The final output is visualized by an LFA strip that is passed externally through the mask. $\mathbf{b}$, Photographs of the SARS-CoV-2 sensor integrated into a face mask. An A-version sensor is shown. c, Key steps of the freeze-dried reactions, each separated by a PVA time delay. Lysis first releases SARS-CoV-2 vRNA, RT-RPA next targets the $\mathrm{S}$ gene for signal amplification at room temperature and finally Cas12a detection of the RPA amplicons results in collateral cleavage of FAM-Biotin ssDNA probes. The reaction flows into the LFA where the visual band pattern formation is dependent upon probe cleavage. d, Sensitivity of the A-version face-mask sensors at various inputs on the sensor zone of IVT-generated SARS-CoV-2 S-gene RNA. The limit of detection threshold, +3 s.d. of the no-template control (NTC), is shown as a red dotted line. e, Representative images of LFA outputs from the sensitivity measurements. f, Specificity demonstration of A-version face-mask sensors shows no cross-reactivity with IVT RNA from other commonly circulating HCoVs. SARS-CoV-2 RNA was added at 100,000 copies. All other HCoV RNAs were tested at 1,000,000 copies. g, Representative images of LFA outputs from the specificity measurements. $\mathbf{h}$, Breath emission simulator setup consisting of a lung simulator for spontaneous breathing generation, a vibrating-mesh nebulizer for aerosol generation and a high-fidelity airway simulator for anatomically precise air flow to the face mask. A B-version face-mask sensor is shown on the simulator. $\mathbf{i}$, On-simulator testing of B-version face-mask sensors under conditions simulating physiological respiratory emission of SARS-CoV-2 target and a face-mask microclimate. $\mathbf{j}$, Representative images of LFA outputs from the on-simulator measurements. All plots ( $\mathbf{d}, \mathbf{f}, \mathbf{i})$ show quantified LFA test band to control band intensity ratios as mean \pm s.d. from $n=4$ independently fabricated and tested face-mask sensors for each of the indicated conditions. AuNP, gold nanoparticle. 
human airway model (Fig. 4h and Supplementary Fig. 16). The target RNA was nebulized to replicate lung emissions with aerosol diameters matching those naturally occurring in breath exhalation

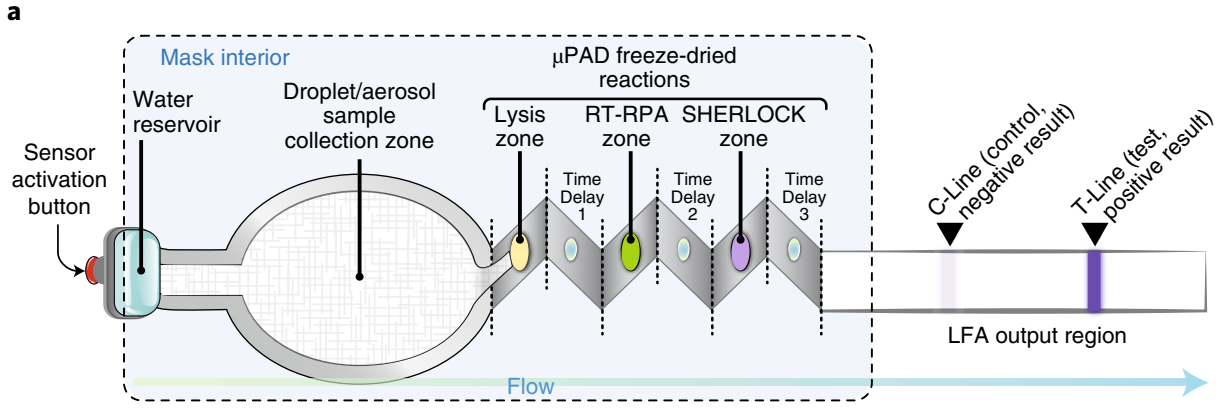

C

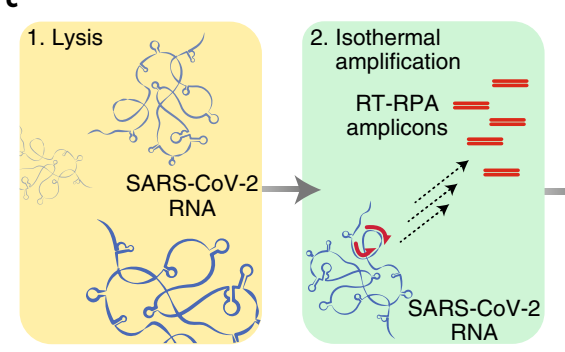

3. Cas12a SHERLOCK

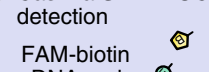

FAM-biotin
ssDNA probe $\odot$ (ब)

cleavage, 田
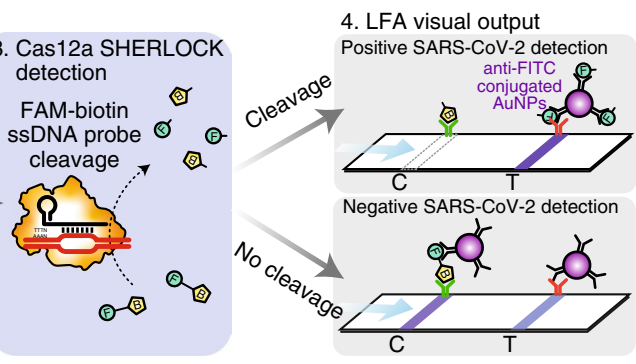

4. LFA visual output

anti-FITC 0 -
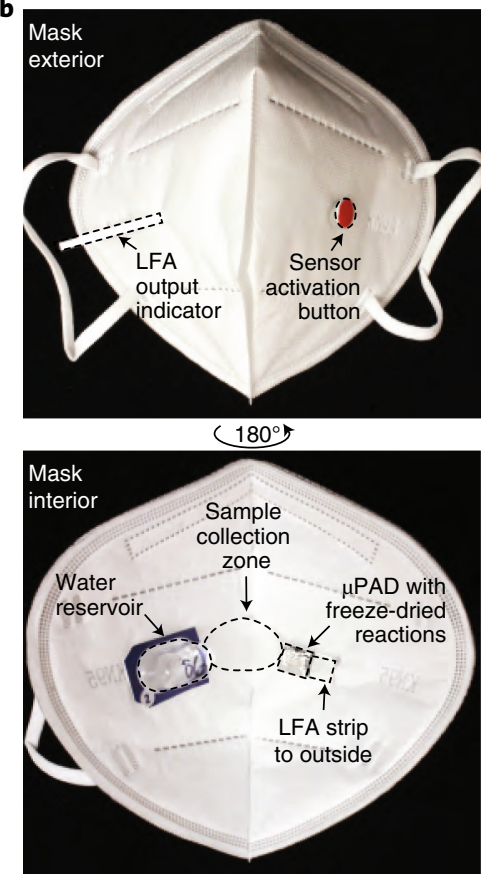

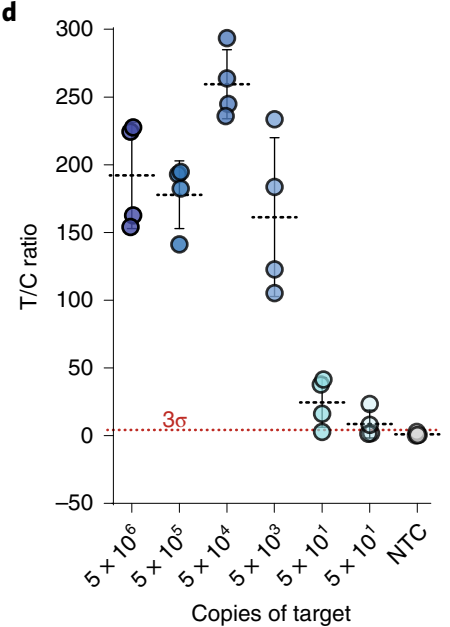

e f

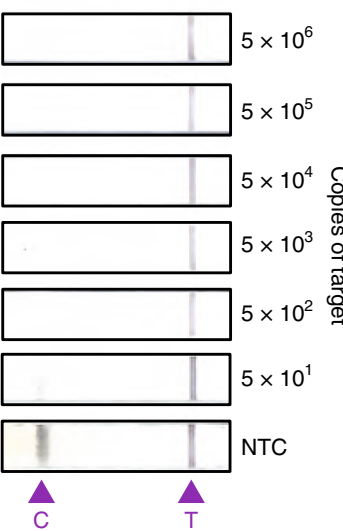

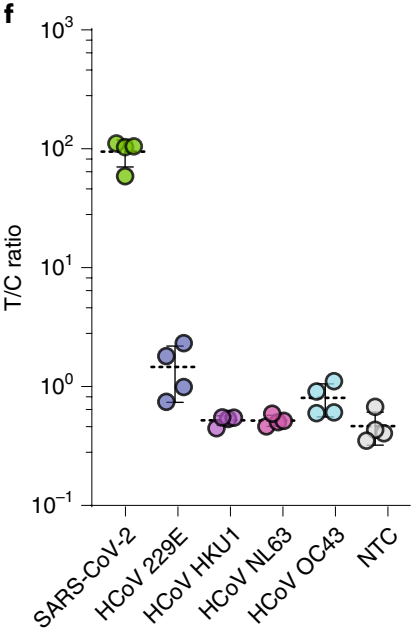
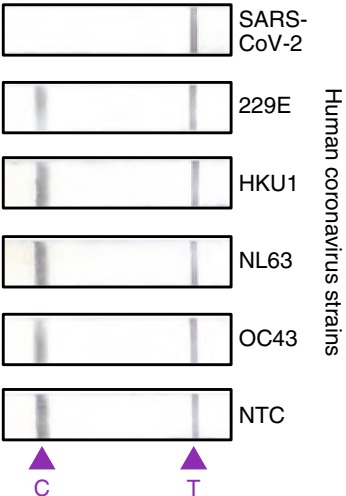

h

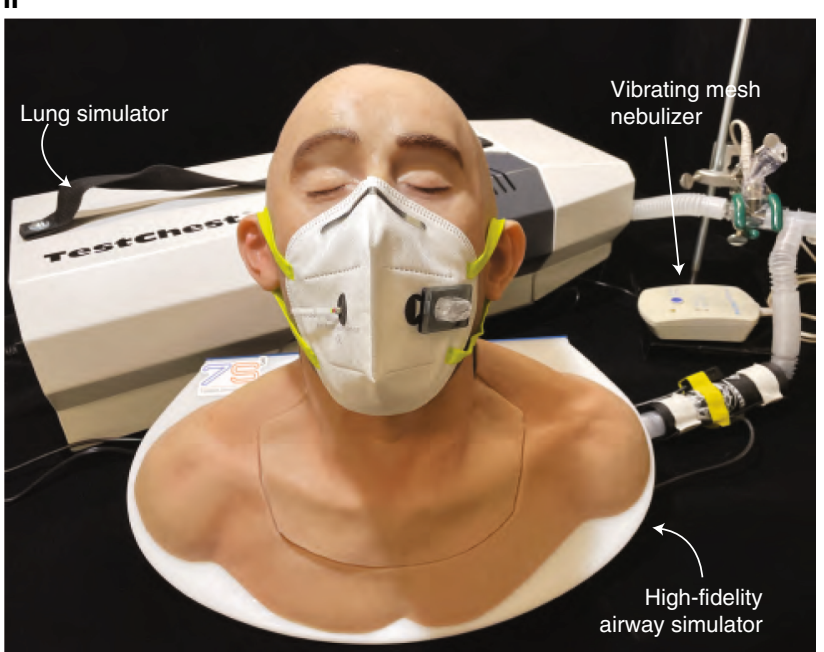

i

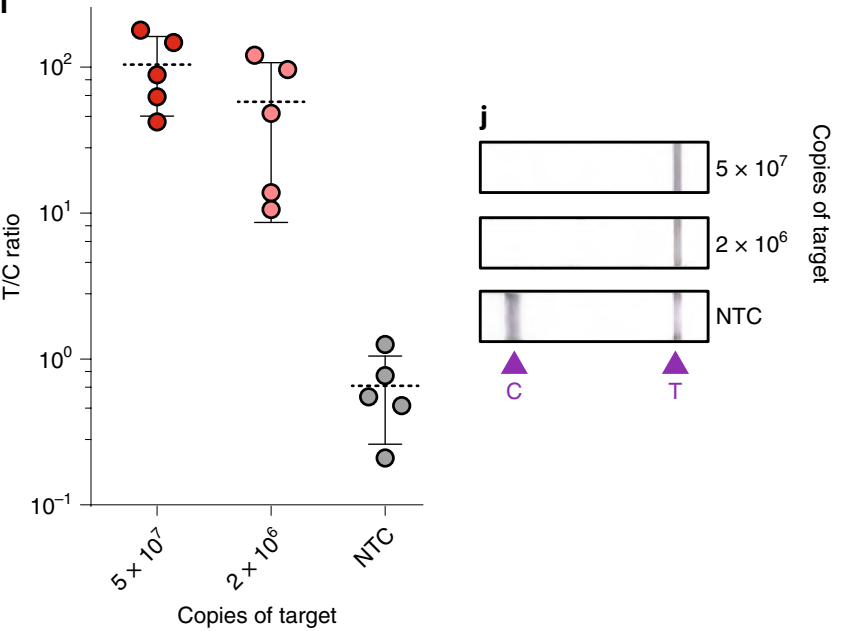


face-mask sensor was able to detect a contrived SARS-CoV-2 viral RNA (vRNA) fragment after a breath sample collection period of $30 \mathrm{~min}$, with a calculated accumulation of $10^{6}-10^{7}$ vRNA copies on the sample pad, as determined by qPCR with reverse transcription (RT-qPCR) (Fig. 4i,j). Clinical measurements have previously shown that the SARS-CoV-2 breath emission rate of infected patients could reach an output $10^{3}-10^{5}$ copies per min (ref. ${ }^{35}$ ).

Unlike other current nucleic acid tests that require laboratory equipment and trained technicians ${ }^{41-45}$, our SARS-CoV-2 face-mask sensor requires no power source, operates autonomously without liquid handling, is shelf-stable, functions at near-ambient temperatures, provides a visual output in under $2 \mathrm{~h}$ and is only $\sim 3 \mathrm{~g}$ in weight. All the user has to do is press a button to activate a reservoir containing nuclease-free water. To our knowledge, no other SARS-CoV-2 nucleic acid test achieves high sensitivity and specificity while operating fully at ambient temperatures, allowing integration into a wearable format. We believe our SARS-CoV-2 mask could combine protection and sensing into one system and could be adapted to discriminate between SARS-CoV-2 and other respiratory viruses, as well as different emerging SARS-CoV-2 variants ${ }^{46,47}$.

\section{Discussion}

We view the wFDCF platform as being complementary to cell-based synthetic biology sensors. We have shown that it enables wearable biosensors that are shelf-stable, genetically programmable and highly sensitive. However, the current wFDCF technology does have a number of limitations, including the single-use nature of the sensors and inability to operate in particular environmental conditions, such as high humidity or underwater. These challenges are also shared by other sensors in which operation requires open access to the environment and will require further engineering to surmount. Our wFDCF sensors are responsive to external rehydration events, such as splashes with contaminated fluids, and withstand inhibitory evaporative and dilutive effects in open-environment conditions $\left(30-40 \%\right.$ relative humidity and $\left.\sim 25-30^{\circ} \mathrm{C}\right)$. Alternatively, user-generated samples such as breath emissions can be used if an on-demand hydration system is employed, as we demonstrate for the SARS-CoV-2-sensing face mask. We showed that these freeze-dried systems generate measurable colorimetric, fluorescence or luminescence outputs upon exposure to relevant real-world targets such as MRSA, Ebola virus or SARS-CoV-2 virus. In the wFDCF POF sensors, continuous monitoring enables rapid alert to an exposure event. We also demonstrated the integration of our device designs into garments that are compatible with wireless sensor networks to provide real-time dynamic monitoring of exposure using custom smartphone applications. Although laboratory testing may be more sensitive, our wFDCF sensors have the advantages of a wearable format, autonomous functioning and rapid results.

To our knowledge, no previous wearable technology has detected viral or bacterial nucleic acid signatures in fluid samples with sensitivities rivaling those of traditional laboratory tests at ambient temperatures. Compared with point-of-care diagnostics, which similarly attempt to eliminate time-consuming and resource-intensive laboratory tests, our sensors do not require the manual application of a swabbed or directly applied sample to provide a readout. They accomplish field sensing on the surface of the user or on surfaces exposed to patient samples, such as the inside of a face mask. Moreover, in contrast to batch-mode point-of-care sensors, they can be networked to provide spatial sensing arrays of lyophilized reactions and lightweight polymer fabrics, thus cloaking the user and continuously generating high-density, real-time outputs without sacrificing comfort or agility in the field. They are designed to operate autonomously and do not require training for use or multiple operations by the user to acquire results, which removes the need to perform regular exposure checks. In contrast to wearable sensors that primarily employ electronic devices to monitor physiological signals such as heart rate or blood oxygen levels, our sensors detect environmental threats or pathogens through nucleic acids, proteins or small molecules. Although electrochemical sensors have been integrated into a wearable format ${ }^{31,48}$, they only detect chemicals, and an easily programmable wearable form for sensitive nucleic acid detection does not currently exist. Integration of our wearable synthetic biology reactions with these advances in electrochemical devices could be a fertile area for expanding the functionality of wearable sensors ${ }^{31,49}$. We have also shown that the sensors can be highly modular and adapted to various form factors, such as clothing or face masks. The key functional differences of our platform over current related technologies, including traditional bench-top assays, are summarized in Supplementary Table 4.

Potential field applications for our wFDCF sensors include warfighters and first responders operating in environments where a specific chemical or biological threat is suspected, and clinicians, health workers and researchers working in high-risk areas. For example, wFDCF-enabled coats and gowns in hospitals could provide alerts to prevent the spread of nosocomial infections. An additional promising application is patient-worn, sensor-enabled personal protective equipment, such as the SARS-CoV-2 face mask.

\section{Online content}

Any methods, additional references, Nature Research reporting summaries, source data, extended data, supplementary information, acknowledgements, peer review information; details of author contributions and competing interests; and statements of data and code availability are available at https://doi.org/10.1038/ s41587-021-00950-3.

Received: 8 November 2019; Accepted: 10 May 2021; Published online: 28 June 2021

\section{References}

1. Khalil, A. S. \& Collins, J. J. Synthetic biology: applications come of age. Nat. Rev. Genet. 11, 367-379 (2010).

2. Tao, X. Smart Fibres, Fabrics and Clothing: Fundamentals and Applications (Elsevier, 2001).

3. Liu, X. et al. Stretchable living materials and devices with hydrogel-elastomer hybrids hosting programmed cells. Proc. Natl Acad. Sci. USA 114, 2200-2205 (2017).

4. Liu, X. et al. 3D printing of living responsive materials and devices. Adv. Mater. 30, 1704821 (2018).

5. Moser, F., Tham, E., González, L. M., Lu, T. K. \& Voigt, C. A. Light-controlled, high-resolution patterning of living engineered bacteria onto textiles, ceramics, and plastic. Adv. Func. Mater. 29, 1901788 (2019).

6. Wang, W. et al. Harnessing the hygroscopic and biofluorescent behaviors of genetically tractable microbial cells to design biohybrid wearables. Sci. Adv. 3, e1601984 (2017)

7. Slomovic, S., Pardee, K. \& Collins, J. J. Synthetic biology devices for in vitro and in vivo diagnostics. Proc. Natl Acad. Sci. USA 112, 14429-14435 (2015).

8. Pardee, K. et al. Paper-based synthetic gene networks. Cell 159, 940-954 (2014)

9. Gootenberg, J. S. et al. Nucleic acid detection with CRISPR-Cas13a/C2c2. Science 356, 438-442 (2017).

10. English, M. A. et al. Programmable CRISPR-responsive smart materials. Science 365, 780-785 (2019).

11. Pardee, K. et al. Portable, on-demand biomolecular manufacturing. Cell 167, 248-259 e212 (2016).

12. Huang, A. et al. BioBits Explorer: a modular synthetic biology education kit. Sci. Adv. 4, eaat5105 (2018).

13. Stark, J. C. et al. BioBits Bright: a fluorescent synthetic biology education kit. Sci. Adv. 4, eaat5107 (2018).

14. Stark, J. C. et al. BioBits Health: classroom activities exploring engineering, biology, and human health with fluorescent readouts. ACS Synth. Biol. 8, 1001-1009 (2019).

15. Choi, J. K., Miki, K., Sagawa, S. \& Shiraki, K. Evaluation of mean skin temperature formulas by infrared thermography. Int. J. Biometeorol. 41, 68-75 (1997).

16. Filonov, G. S., Kam, C. W., Song, W. \& Jaffrey, S. R. In-gel imaging of RNA processing using broccoli reveals optimal aptamer expression strategies. Chem. Biol. 22, 649-660 (2015). 
17. Hall, M. P. et al. Engineered luciferase reporter from a deep sea shrimp utilizing a novel imidazopyrazinone substrate. ACS Chem. Biol. 7, 1848-1857 (2012).

18. Hayes' Handbook of Pesticide Toxicology (ed. Krieger, R.) (Academic Press, 2010).

19. Chen, J. S. et al. CRISPR-Cas12a target binding unleashes indiscriminate single-stranded DNase activity. Science 360, 436-439 (2018).

20. Gootenberg, J. S. et al. Multiplexed and portable nucleic acid detection platform with Cas13, Cas12a, and Csm6. Science 360, 439-444 (2018).

21. Li, S.-Y. et al. CRISPR-Cas12a has both cis- and trans-cleavage activities on single-stranded DNA. Cell Res. 28, 491 (2018).

22. Piepenburg, O., Williams, C. H., Stemple, D. L. \& Armes, N. A. DNA detection using recombination proteins. PLoS Biol. 4, e204 (2006).

23. Lee, A. S. et al. Methicillin-resistant Staphylococcus aureus. Nat. Rev. Dis. Primers 4, 18033 (2018).

24. Falugi, F., Kim, H. K., Missiakas, D. M. \& Schneewind, O. Role of protein A in the evasion of host adaptive immune responses by Staphylococcus aureus. mBio 4, e00575-00513 (2013).

25. Westh, H., Hougaard, D. M., Vuust, J. \& Rosdahl, V. T. Prevalence of erm gene classes in erythromycin-resistant Staphylococcus aureus strains isolated between 1959 and 1988. Antimicrob. Agents Chemother. 39, 369-373 (1995).

26. Alieva, N. O. et al. Diversity and evolution of coral fluorescent proteins PLoS ONE 3, e2680 (2008).

27. Shaner, N. C. et al. Improved monomeric red, orange and yellow fluorescent proteins derived from Discosoma sp. red fluorescent protein. Nat. Biotechnol. 22, 1567-1572 (2004).

28. Pedelacq, J. D., Cabantous, S., Tran, T., Terwilliger, T. C. \& Waldo, G. S. Engineering and characterization of a superfolder green fluorescent protein. Nat. Biotechnol. 24, 79-88 (2006).

29. Ju, J. et al. A multi-structural and multi-functional integrated fog collection system in cactus. Nat. Commun. 3, 1247 (2012).

30. Gouma, P. I., Wang, L., Simon, S. R. \& Stanacevic, M. Novel isoprene sensor for a flu virus breath monitor. Sensors 17, 199 (2017).

31. Maier, D. et al. Toward continuous monitoring of breath biochemistry: a paper-based wearable sensor for real-time hydrogen peroxide measurement in simulated breath. ACS Sens. 4, 2945-2951 (2019).

32. Gould, O., Ratcliffe, N., Krol, E. \& de Lacy Costello, B. Breath analysis for detection of viral infection, the current position of the field. J. Breath Res. 14, 041001 (2020).

33. Tromberg, B. J. et al. Rapid scaling up of Covid-19 diagnostic testing in the United States-the NIH RADx initiative. N. Engl. J. Med. 383, 1071-1077 (2020)
34. Liu, Y. et al. Aerodynamic analysis of SARS-CoV-2 in two Wuhan hospitals. Nature 582, 557-560 (2020).

35. Ma, J. et al. Exhaled breath is a significant source of SARS-CoV-2 emission. Preprint at medRxiv https://doi.org/10.1101/2020.05.31.20115154 (2020).

36. Leung, N. H. L. et al. Respiratory virus shedding in exhaled breath and efficacy of face masks. Nat. Med. 26, 676-680 (2020).

37. Darnell, M. E., Subbarao, K., Feinstone, S. M. \& Taylor, D. R. Inactivation of the coronavirus that induces severe acute respiratory syndrome, SARS-CoV. J. Virol. Methods 121, 85-91 (2004).

38. Shen, S. et al. The severe acute respiratory syndrome coronavirus $3 \mathrm{a}$ is a novel structural protein. Biochem. Biophys. Res. Commun. 330, 286-292 (2005).

39. Gui, M. et al. Electron microscopy studies of the coronavirus ribonucleoprotein complex. Protein Cell 8, 219-224 (2017).

40. Vogels, C. B. F. et al. Analytical sensitivity and efficiency comparisons of SARS-CoV-2 RT-qPCR primer-probe sets. Nat. Microbiol. 5, 1299-1305 (2020).

41. Baek, Y. H. et al. Development of a reverse transcription-loop-mediated isothermal amplification as a rapid early-detection method for novel SARS-CoV-2. Emerg. Microbes Infect. 9, 998-1007 (2020).

42. Joung, J. et al. Point-of-care testing for COVID-19 using SHERLOCK diagnostics. Preprint at medRxiv https://doi.org/10.1101/2020.05.04.20091231 (2020)

43. Arizti-Sanz, J. et al. Streamlined inactivation, amplification, and Cas13-based detection of SARS-CoV-2. Nat. Commun. 11, 5921 (2020).

44. Yan, C. et al. Rapid and visual detection of 2019 novel coronavirus (SARS-CoV-2) by a reverse transcription loop-mediated isothermal amplification assay. Clin. Microbiol. Infect. 26, 773-779 (2020).

45. Broughton, J. P. et al. CRISPR-Cas12-based detection of SARS-CoV-2. Nat. Biotechnol. 38, 870-874 (2020).

46. Koyama, T., Weeraratne, D., Snowdon, J. L. \& Parida, L. Emergence of drift variants that may affect COVID-19 vaccine development and antibody treatment. Pathogens 9, 324 (2020).

47. Li, Q. et al. The impact of mutations in SARS-CoV-2 spike on viral infectivity and antigenicity. Cell 182, 1284-1294 e1289 (2020).

48. Barfidokht, A. et al. Wearable electrochemical glove-based sensor for rapid and on-site detection of fentanyl. Sens. Actuators B Chem. 296, 126422 (2019).

49. Dincer, C. et al. Disposable sensors in diagnostics, food, and environmental monitoring. Adv. Mater. 31, e1806739 (2019).

Publisher's note Springer Nature remains neutral with regard to jurisdictional claims in published maps and institutional affiliations.

(c) The Author(s), under exclusive licence to Springer Nature America, Inc. 2021 


\section{Methods}

Fabrication of colorimetric synthetic biology wearable modules. Translucent (Fig. 1a, top) and opaque (Fig. 1a, middle/bottom) layers were made using skin-safe Ecoflex silicone elastomer (Smooth-On), precast overnight and laser-cut on a $75 \mathrm{~W}$ Epilog Legend 36EXT, according to the layouts shown in Fig. 1a and Supplementary Fig. 2a. After laser-cutting, the silicone pieces were placed in a warm wash $\left(45^{\circ} \mathrm{C}\right)$ with Tergazyme detergent (Alconox) for $1 \mathrm{~h}$ with agitation, followed by three washes in $18-\Omega$ pure water and a final wash in $70 \%$ ethanol, before allowing them to air-dry. Layers were aligned and bonded together by depositing freshly made, uncured liquid silicone elastomer and post-curing overnight at $65^{\circ} \mathrm{C}$ in a well-ventilated oven to obtain the final assembled prototypes. The final assembled elastomer prototypes were thoroughly sprayed with RNase Away Decontaminant (Thermo Fisher Scientific) and washed with $70 \%$ ethanol twice before being stored in petri dishes.

For the support matrices housing the cell-free reactions, clean Whatman No. 4 filter-papers (GE Healthcare Lifesciences) (Fig. 1a, reaction insert) were punched to obtain cellulose discs with dimensions of $8-\mathrm{mm}$ diameter and $0.5-\mathrm{mm}$ thickness. These discs were incubated overnight in $0.01 \%$ diethyl pyrocarbonate, washed three times with nuclease-free water, then incubated with $5 \%$ BSA (MilliporeSigma) in $50 \mathrm{mM}$ Tris buffer, $\mathrm{pH} 7.5$, for $1 \mathrm{~h}$ with gentle agitation. The prepared BSA-blocked discs were frozen at $-80^{\circ} \mathrm{C}$ and subsequently freeze-dried. These lyophilized BSA-blocked discs were used as a scaffold for the deposition of colorimetric wearable synthetic biology reactions in wFDCF sensors. The saturated reaction discs were finally snap-frozen in liquid nitrogen and freeze-dried for 8-12 h in an SP Scientific Freezemobile lyophilizer (SP Industries).

Freeze-dried reaction discs were then inserted through the wicking ports of the elastomer chambers for assembly. The silicone elastomer chambers in the colorimetric device exhibit three $3 \times 5-\mathrm{mm}^{2}$ curved wicking ports in each of the four wells, which allow inflow routes for fluid entry while delaying evaporation of cell-free reaction (Supplementary Fig. 2a). The device chamber walls were aligned and bonded using uncured elastomer, to prevent flow or lateral diffusion of the reaction after rehydration. The wicking of contaminated fluid through the entry ports is primarily mediated by capillary action. An exposure event leads to rehydration of the reaction disc containing the chosen FDCF system, which marks $t=0$ in the validation experiments (Fig.1d-g). A magnified photograph of an activated reaction well containing an Ebola virus DNA toehold wFDCF sensor is shown in Supplementary Fig. 3a, whereas the activation of a fabricated wearable bracelet using the same system is depicted in Supplementary Fig. 3c. All of the colorimetric wFDCF sensors were tested at $30^{\circ} \mathrm{C}$ and ambient humidity to simulate surface body temperature.

Preparation of optimized colorimetric wearable synthetic biology reactions. Each colorimetric wFDCF reaction used for lyophilization, assuming a 50- $\mu$ l rehydration volume, was a $75-\mu$ l cell-free NEB PURExpress reaction (New England Biolabs). Thus, each rehydrated reaction is a $\times 1.5$-concentrated cell-free reaction based on the suggested reaction composition indicated by the manufacturer. Each reaction consisted of: $30 \mu \mathrm{l}$ of PURExpress Component A, $22.5 \mu \mathrm{l}$ of PURExpress Component $\mathrm{B}, 0.6 \mathrm{mg} \mathrm{ml}^{-1} \mathrm{CPRG}$ (MilliporeSigma), $76 \mathrm{U}$ of RNase Inhibitor (Roche) and a DNA template encoding the desired artificial genetic circuit at $5 \mathrm{ng}^{-1} \mathrm{l}^{-1}$. For the TetR transcriptional regulation circuit, FPLC-purified recombinant TetR protein was supplemented in the reaction at a concentration of $120 \mu \mathrm{g} \mathrm{ml}^{-1}$. During activation of the various wFDCF reactions by rehydration, pure nuclease-free $\mathrm{H}_{2} \mathrm{O}$ was used for the constitutive $\mathrm{LacZ}$ circuit, $25 \mu \mathrm{g} \mathrm{ml}^{-1}$ anhydrotetracycline (aTc) inducer was used for the TetR-regulated circuit, $300 \mathrm{nM}$ of Ebola viral genome trigger was used for the toehold regulated circuit and $1 \mathrm{mM}$ of theophylline was used for the riboswitch-regulated circuit. The theophylline riboswitch reactions also included 2 -phenylethyl $\beta$-D-thiogalactoside (MilliporeSigma), a $\beta$-galactosidase inhibitor, at a final concentration of $250 \mu \mathrm{M}$ to suppress the background due to leakiness in these genetic circuits. The Ebola RNA genome trigger was acquired by an in vitro transcription reaction utilizing the HiScribe T7 Quick High Yield RNA Synthesis Kit (New England Biolabs), using a DNA template as indicated in Supplementary Table 2. Each wFDCF reaction was applied to a BSA-blocked cellulose disc inserted into a $2-\mathrm{ml}$ microcentrifuge tube. After the reaction was absorbed into the disc, the tubes were submerged in liquid nitrogen to snap-freeze the disc and allowed to lyophilize for $12 \mathrm{~h}$. All of the colorimetric wFDCF sensors were tested at $30^{\circ} \mathrm{C}$ and ambient humidity to simulate surface body temperature. The colorimetric wFDCF reactions presented in this work were from distinct sensors, in which each data point is the intensity value of a defined area of the green channel from the color-deconvolution function in ImageJ. The selected area size was kept constant for all sensors. Each dataset plotted in Fig. 1 is the average of three independently measured wells. Statistical significance values for specific time points were calculated using unpaired parametric Student's $t$-test (two-sided).

Evaporation and dilution experiments in wearable synthetic biology devices. Evaporation tests were performed by cutting $10 \times 10-\mathrm{cm}^{2}$ Whatman No. 4 filter paper squares and performing the cleaning and BSA blocking as described above for the discs. Each square was freeze-dried with $100 \mu$ l of a $1 \times$ PURExpress cell-free reaction with CPRG substrate and a constitutive LacZ plasmid. Various temperature $\left(27-32^{\circ} \mathrm{C}\right)$ and fluid exposure conditions were investigated in combination with different coverage ratios of the rehydrated test squares to assess evaporation reduction. Suitable activity of the rehydrated reactions was assessed by visual inspection of the conversion of the colorimetric substrate from yellow to purple. The port designs were selected empirically due to suitable activation of synthetic biology reactions with reduced evaporation rates $(<20 \%$ of initial fluid volume in $2 \mathrm{~h}$ ) at $30-40 \%$ relative humidity.

\section{Kinetic enhancement by freeze-dried concentration of cell-free reaction} components. Optimization testing of cell-free component concentrations on the kinetics of the reactions was performed by assembling PURExpress systems, according to the manufacturer's specifications, at various volumes $\left(V_{\text {initial }}\right)$ and then lyophilizing the reactions in PCR tubes overnight (Extended Data Fig. 1a). Next, the lyophilized pellets were rehydrated using the same sample volume $\left(V_{\text {final }}\right)$, so that the tested fold-concentration was $\left(V_{\text {initial }} / V_{\text {final }}\right)$. PURExpress concentrations ranging from $\times 1$ to $\times 2.5$ were tested in replicate by incubation of $10-\mu 1$ reactions at $30^{\circ} \mathrm{C}$ for up to $90 \mathrm{~min}$, followed by photographic imaging of the colorimetric changes (Extended Data Fig. 1b) and absorbance measurements at $570 \mathrm{~nm}$ (Extended Data Fig. 1c). The time to half-maximal output signal for each base or concentrated reaction (Extended Data Fig. 1d) was calculated by a least square fitting of the acquired data.

Screening of textiles for FDCF synthetic biology reactions. General compatibility of different textiles to FDCF synthetic biology reactions was tested in 103 different fabric materials (for example, silks, cotton, rayon, linen, hemp, bamboo, wool, polyester, polyamide, nylon and combination threads) under activation conditions (Supplementary Table 1 and Supplementary Fig. 4). A detailed list of the textiles used for this substrate screening can be found in Supplementary Table 1. This compatibility of these textiles to FDCF synthetic biology reactions was compared with samples using Whatman No. 4 filter paper (GE Healthcare Lifesciences) and samples in liquid form without any substrate as seen in Supplementary Fig. 5. All tests used a T7RNAP-regulated LacZ circuit for constitutive expression. For this evaluation, fabric samples were identified and cut into $2 \times 2-\mathrm{cm}^{2}$ squares. Visible particles were removed from the fabrics using an adhesive roller. All fabric squares were cut into $1 \times 2-\mathrm{cm}^{2}$ pairs and washed thoroughly within $1.5-\mathrm{ml}$ Eppendorf tubes with $1 \mathrm{ml}$ of dd- $\mathrm{H}_{2} \mathrm{O}$ for $30 \mathrm{~min}$, floating in a sonication bath at $80^{\circ} \mathrm{C}$. The washed samples were left to cool to room temperature and then washed with running dd- $\mathrm{H}_{2} \mathrm{O}$ for $10 \mathrm{~s}$. One of each pair of fabric square types was placed in $1.25 \mathrm{ml}$ of a $5 \%$ BSA solution for $12 \mathrm{~h}$. After BSA incubation, the treated fabrics were cleaned with running dd- $\mathrm{H}_{2} \mathrm{O}$ for $10 \mathrm{~s}$. BSA-blocked and unblocked samples were then placed into fresh Eppendorf tubes with holes in the caps to allow for overnight desiccation of the fabrics at $60^{\circ} \mathrm{C}$. Dried BSA-blocked and unblocked fabrics were then cut in triplicate with clean 2-mm-diameter disc biopsy punchers and placed in their respective slots in flat 384-well black polystyrene plates with a clear glass bottoms (Corning, ref. no. 3544) for testing. Cell-free PURExpress in vitro protein synthesis solution (New England Biolabs) was combined with a constitutive LacZ template containing $0.6 \mathrm{mg} \mathrm{ml}^{-1} \mathrm{CPRG}$ and spotted $(1.8 \mu \mathrm{l})$ on each of the fabric wells. Control wells containing $2-\mathrm{mm}$ discs of Whatman No. 4 filter paper were also filled with 1.8 - $\mu$ l constitutive LacZ test reactions, whereas $7 \mu \mathrm{l}$ was spotted on empty wells as liquid controls. A transparent adhesive PCR cover compatible with freezing was then placed over the plate and pressed with a roller to seal chambers. A small opening was pierced in each well with a 25 -gauge $\times 5 / 8$ $(0.5 \mathrm{~mm} \times 16 \mathrm{~mm})$ BD PrecisionGlide Needle (Becton, Dickinson and Company, ref. no. 305122) to allow for sublimation during lyophilization. Prepared plates were wholly immersed into liquid nitrogen for $1 \mathrm{~min}$. A chilled metallic plate (maintained at $-80^{\circ} \mathrm{C}$ with dry ice) was immediately put in contact with the bottom of the scored plates with the sealed frozen samples. A single $15^{\prime \prime} \times 17^{\prime \prime}$ Kimwipe (Kimtech, Kimberly-Clark) was placed on top of the plate humidity openings. Then the 384-well test plate with top Kimwipe and the bottom metallic chiller were wrapped with three layers of aluminium foil. The entire wrapped bundle was then placed inside a sealed glass lyophilization chamber and connected to the freeze-drying machine. Lyophilization was performed for $2 \mathrm{~h}$. Freeze-dried paper samples were rehydrated with $\mathrm{dd}-\mathrm{H}_{2} \mathrm{O}$ to the original reaction volume. The colorimetric change was measured after overnight incubation $(12 \mathrm{~h})$ at $37^{\circ} \mathrm{C}$ using a BioTek NEO HTS plate reader (BioTek Instruments) in kinetic absorbance readout mode (Supplementary Fig. 5). Best observed functionality, as measured by the aggregated score shown in Supplementary Fig. 6, was achieved using a fabric with $85 \%$ polyester and $15 \%$ polyamide fibers. This substrate was used for all further fluorescence and luminescence experiments, except for the case for a fluorescence Zika DNA toehold sensing reaction (Supplementary Fig. 7), which was also tested on a $100 \%$ mercerized cotton thread to validate the possibility of running FDCF reactions at the single-fiber level with this natural material commonly used in wound care.

Fabrication of fluorescence/luminescence synthetic biology wearable textile module. After screening of compatible textiles for FDCF synthetic biology reactions, the best-performing hydrophilic textile substrate ( $85 \%$ polyester/ $15 \%$ polyamide) was used as weft for a textile inter-woven with a warp made of inert flexible POFs and polyester support threads. Such POFs were used for distributed optical interrogation of fluorescent or luminescent synthetic biology reactions 
within this fabric (three fibers per well). POFs were weaved into this hydrophilic combination fabric using a standard industrial loom (DREAMLUX, Samsara S.r.l.), according to the design presented in Supplementary Fig. 8. Once fabric samples were manufactured, three-strip arrangements of this hydrophilic POF fabric were cut to fit the device and laser-etched $(5 \mathrm{~mm})$ to disrupt the cladding in the POFs sections within the reaction zones (Extended Data Fig. 2a-e). Black elastomer layers (top and bottom in Extended Data Fig. 2b) were precast overnight and laser-cut according to the layout shown in Extended Data Fig. 2b,e. The silicone elastomer chambers in this device exhibit two $3 \times 5-\mathrm{mm}^{2}$ curved wicking ports that allow for fluid entry while still delaying evaporation within reaction fabric. Uncured black silicone elastomer was stamp-patterned onto the precast layers as well as into the internal POF fabric strips to be aligned and assembled, preventing air bubble formation between device layers and elastomer wicking in reaction zones. Final assembly of the base three-well sensor 'patch' can be seen in Extended Data Fig. 2b,f,g. Devices were then placed under vacuum for $15 \mathrm{~min}$ to remove bubbles and were allowed to cure overnight at $65^{\circ} \mathrm{C}$. As with the colorimetric prototypes, the fluorescent POF prototypes were thoroughly sprayed with RNase Away Decontaminant (Thermo Fisher Scientific) and washed with 70\% ethanol twice before being stored in petri dishes. Once the assembled device was fully cured, POF fibers were separated into excitation and emission bundles and then covered with blackout adhesive fabric as well as black heat shrink tubing $(6 \mathrm{~mm})$ to prevent environmental light leakage. Blackout fabric discs $(10 \mathrm{~mm})$ made of a black polyester knit item (no: 322323, MoodFabrics) were soaked in RNase Away Decontaminant for $5 \mathrm{~min}$, and washed thoroughly with $70 \%$ ethanol followed by water. The washed blackout fabric was incubated in $0.1 \%$ Triton X-100 for $5 \mathrm{~min}$ (as a wetting agent to enhance the ability of the textile to absorb water) and then excess solution was removed and the fabric pieces allowed to air-dry. The final blackout fabric discs were placed inside the reaction chamber with tweezers to aid in environmental light-blocking over sensing fibers. Finally, quick-turn stainless steel coupling sockets (no. 5194K42, McMaster-Carr) were added to the ends of the sensor device bundles for connection with the wearable spectrometer. The finalized wFDCF sensor device can be seen in Extended Data Fig. 2f,g

\section{Hardware/software implementation of wearable POF spectrometer. A} custom-made wearable spectrometer with internal processing and wireless connectivity modules was fabricated to provide unsupervised sensing of on-body synthetic biology reactions (Extended Data Fig. 8). The device electronics were based on a Raspberry Pi Zero W v.1.3 architecture (Raspberry Pi Foundation) with connection to a custom shield for battery power, an environmental sensing module, a light-emitting diode (LED) illumination module and a flexible camera for imaging (Extended Data Fig. 8a). The Raspberry Pi Zero W was selected as the microprocessor for this application due to its low cost $(<\mathrm{US} \$ 15.00)$, small profile/weight $\left(65 \times 30 \times 5 \mathrm{~mm}^{3} / 12 \mathrm{~g}\right)$, high performance $(1 \mathrm{GHz}$ single-core ARM1176JZF-S CPU, 512 MB RAM, VideoCore IV GPU) and on-board wireless connectivity (802.11 b/g/n LAN, Bluetooth(R) 4.1, Bluetooth Low Energy). Regulated battery power was achieved using a PiZ-UpTime module, which is an uninterruptible power supply shield for Raspberry Pi Zero (Alchemy Power) that uses a rechargeable lithium-ion 14500 battery (battery and power management in Extended Data Fig. 8a) to reliably provide the charge capacity for $48 \mathrm{~h}$ of intermittent device operation, continuously collecting data at a frequency of one measurement per minute. In-device sensing of temperature, humidity, atmospheric pressure, altitude, total volatile organic compound and equivalent $\mathrm{CO}_{2}$ was achieved using an I2C Environmental CCS811/BME280 Qwiic-Breakout (SparkFun Electronics). The POF illumination module was achieved using a Saber Z4 Luxeon Z $20 \mathrm{~mm}$ Square Quad Color Mixing Array LED Module with aluminium base (Quadica Developments, Luxeon) connected to a 12-Channel 16-Bit PWM TLC59711 LED Driver with SPI Interface (Adafruit Industries). Four Luxeon Star LEDs were installed in the device with wavelengths $447 \mathrm{~nm}$, $470 \mathrm{~nm}, 505 \mathrm{~nm}$ and 6,500 K white (LEDs and driver in Extended Data Fig. 8a) An $8.6 \times 8.6 \mathrm{~mm}^{2}$ Zero Spy Camera with 2" cable (Raspberry Pi Foundation) was connected to the Raspberry Pi Zero W using a flat serial interphase connector to provide POF imaging capabilities to the device. A single 5-mm Infinite aspherical plastic collimator (part no.: 191-66041G, Quarton), with numerical aperture 0.27 and effective focal length $4.96 \mathrm{~mm}$, was placed on top of the camera to allow for magnified POF imaging in proximity to the camera. The wearable spectrometer was covered by a two-part case fabricated using black photoreactive resin and a stereolithography three-dimensional printing method using a Form 2 printer (Formlabs) as seen in Extended Data Fig. 8a. A view of the open device is shown in Extended Data Fig. 8b, while a closed view is shown in Extended Data Fig. 8c. This case included geometrical features to fit and align the camera/lens arrangement and the removable 3-mm-diameter amber acrylic filter for fluorescence readings (slot arrangement in Extended Data Fig. 8d). Also, the case features a slot for the four-LED arrangement and a vent for the environmental sensors, as well as female Luer connection-to-fitting quick-turn stainless steel coupling sockets (no. 5194K42, McMaster-Carr). A top view of the assembled wearable POF spectrometer is shown in Extended Data Fig. 8e, while the integration of this device within a wearable garment with wFDCF sensors is shown in Extended Data Fig. 8f. The final volume of our wearable spectrometer device was approximately $235 \mathrm{~cm}^{3}$ with a total weight of around $173.8 \mathrm{~g}$ (6.13 ounces), with a total cost of material and consumable supplies under US\$100. Base data-collection software (test version) implemented in Python for control of the Raspberry Pi Zero $\mathrm{W}$ within the wearable POF spectrometer is also provided as part of the Supplementary Information.

Preparation of optimized fluorescence wearable synthetic biology reactions. Constitutive sfGFP expression reactions for wFDCF testing (Fig. 2c) were prepared by combining $50 \mu \mathrm{l}$ of $1 \times \mathrm{NEB}$ cell-free PURExpress in vitro protein synthesis solution with $0.5 \%$ Roche Protector RNase Inhibitor and $10 \mathrm{ng}^{-1} \mathrm{l}^{-1}$ constitutive $\mathrm{P}_{\mathrm{T} 7}$-sfGFP plasmid (+) or without as controls (-). Prepared reactions were quickly deposited in-fabric to be snap-frozen and then lyophilized for 4-8h within the device. Activation of sensors was achieved by rehydration with a fluid splash of dd- $\mathrm{H}_{2} \mathrm{O}$.

The theophylline riboswitch sensor reactions for wFDCF testing (Fig. 2d) were prepared using $1 \times$ NEB cell-free PURExpress supplemented with $10 \mathrm{ng}^{-1} \mathrm{l}^{-1}$ theophylline riboswitch sensor E mRNA in dd- $\mathrm{H}_{2} \mathrm{O}$. The prepared sensor reactions $(50 \mu \mathrm{l}$ per well) were quickly deposited in-fabric, snap-frozen in liquid nitrogen and then lyophilized for $4-8 \mathrm{~h}$ within the device. Activation of sensors was achieved by rehydration with a fluid splash of dd $-\mathrm{H}_{2} \mathrm{O}$ spiked with $1 \mathrm{mM}$ theophylline for the positive samples, while $0 \mathrm{mM}$ theophylline was used for controls.

Dimeric Broccoli fluorescent aptamer sensor reactions for wFDCF testing (Fig. 2e) were prepared using $1.5 \times$ NEB cell-free PURExpress with $25 \mathrm{ng}^{-1}$ pJL1-F30-2xd-Broccoli aptamer DNA in dd- $\mathrm{H}_{2} \mathrm{O}$. Prepared sensor reactions $(50 \mu \mathrm{l}$ per well) were quickly deposited in-fabric to be snap-frozen and then lyophilized for $4-8 \mathrm{~h}$ within the device. Activation of sensors was achieved by rehydration with a fluid splash of dd $-\mathrm{H}_{2} \mathrm{O}$ spiked with $50 \mu \mathrm{M}$ DFHBI-1T (Tocris Bioscience) substrate for the positive samples, while $0 \mu \mathrm{M}$ DFHBI-1T substrate was used for controls.

Zika RNA toehold switch sensor reactions for wFDCF testing (Supplementary Fig. 7) were prepared using $1 \times$ NEB cell-free PURExpress with 33 nM Zika DNA toehold sensor $27 \mathrm{~B}$ in dd- $\mathrm{H}_{2} \mathrm{O}$. Prepared sensor reactions were quickly deposited in a mercerized cotton thread or paper samples to be snap-frozen and then lyophilized for $4-8 \mathrm{~h}$ within a 384-well plate. Activation of sensors was achieved by rehydration with dd $-\mathrm{H}_{2} \mathrm{O}$ spiked with $2 \mu \mathrm{M}$ freshly made Zika trigger RNA for the positive samples, while $0 \mu \mathrm{M}$ Zika trigger RNA was used for controls.

For the wearable nerve agent sensor experiments (Fig. $2 \mathrm{~g}$ ), $50-\mu \mathrm{l}$ reactions consisting of $0.5 \mathrm{U} \mathrm{ml}^{-1} \mathrm{AChE}$ (Type $\mathrm{V}-\mathrm{S}$ from Electrophorus electricus,

MilliporeSigma), $0.1 \mathrm{U} \mathrm{ml}^{-1}$ of choline oxidase (recombinant Arthrobacter sp., MilliporeSigma), $0.1 \mathrm{mg} \mathrm{ml}^{-1}$ of freshly prepared horseradish peroxidase (Type VI, MilliporeSigma) and $125 \mu \mathrm{M}$ of the fluorescent reporter substrate Amplite-IR (AAT Bioquest) in a final buffer of $10 \mathrm{mM}$ HEPES, $\mathrm{pH} 8.0,1 \mathrm{mg} \mathrm{ml}^{-1} \mathrm{BSA}, 1 \%$ fish gelatin and $5 \%$ trehalose. The reactions were applied to two Whatman No. 4 filter paper $0.8-\mathrm{cm}$ discs, snap-frozen in liquid nitrogen and lyophilized for at least $12 \mathrm{~h}$. To test in the fluorescent wearable prototype, the paper discs containing the freeze-dried reactions were inserted into the wearable devices and rehydrated with $75 \mu \mathrm{l}$ of $50 \mu \mathrm{M}$ acetylcholine (MilliporeSigma) with or without the nerve agent paraoxon-ethyl (MilliporeSigma). The fluorescent wearable device for the nerve agent was altered for the detection of NIR fluorescence by replacing the optical components with excitation using a 627-nm red quad-LED array module (Quadica Developments, Luxeon). Additionally, the emission camera was substituted with a NoIR Zero Spy Camera without infrared filter, on top of which we positioned three gel transmission filters (no. 381, no. 382 and no. 383; Rosco Laboratories) to form a dedicated emission filtering stack with $<1 \%$ cutoff at $660 \mathrm{~nm}$ and peak transmittance at $740 \mathrm{~nm}$. All of the fluorescent wFDCF sensors were tested at $30^{\circ} \mathrm{C}$ and ambient humidity to simulate surface body temperature. All fluorescent wFDCF data presented in this work were from distinct sensors, in which each data point is the integrated value of color-deconvoluted optical fiber signals from one sensor, using the green channel for fluorescence and the blue channel for luminescence. Any fiber optic signals that were 1 s.d. below the mean of all fibers combined were removed from the analysis. All of the cell-free and enzymatic wFDCF sensor plots are the average of three independent wells with each well containing three separate fiber optic sensors, for a total of nine fiber outputs presented per variable. Statistical significance values for specific time points were calculated using unpaired parametric Student's $t$-test (one-sided).

\section{Preparation of optimized luminescence wearable synthetic biology reactions.}

HIV RNA toehold switch sensor reactions for luminescence wFDCF testing (Fig. $2 \mathrm{f}$ and Extended Data Fig. 3a) were prepared in 50 - $\mu$ l batches using $20 \mu \mathrm{l}$ of NEB cell-free PURExpress Component A, $15 \mu \mathrm{l}$ of NEB Component B, $2.5 \mu \mathrm{l}$ of murine RNase inhibitor (New England Biolabs), $6 \mathrm{ng}^{-1} \mathrm{HIV}$ toehold sensor template with an nLuc output and $0.5 \mu$ l of luciferin substrate (Promega) in dd- $\mathrm{H}_{2} \mathrm{O}$. Prepared sensor reactions $(50 \mu \mathrm{l}$ per well) were quickly deposited in-fabric to be snap-frozen and then lyophilized for $4-8 \mathrm{~h}$ within the device. Activation of sensors was achieved by rehydration with a fluid splash of dd- $\mathrm{H}_{2} \mathrm{O}$ spiked with $10 \mu \mathrm{M}$ HIV trigger RNA freshly made for the positive samples, while $0 \mu \mathrm{M}$ HIV trigger RNA was used for controls. The constitutive nLuc control reaction performed in singlicate shown as reference for Extended Data Fig. 3b was performed similarly but substituting the toehold switch with a plasmid with an nLuc operon regulated by a T7 promoter.

B. burgdorferi RNA Lyme disease toehold switch sensor reactions for luminescence wFDCF testing (Extended Data Fig. 3b) were prepared in 50- $\mu$ l 
batches using $20 \mu \mathrm{l}$ of NEB cell-free PURExpress solution A, $15 \mu \mathrm{l}$ of solution B, $2.5 \mu \mathrm{l}$ of murine RNase inhibitor, $18 \mathrm{nM}$ B. burgdorferi toehold DNA with luciferase operon and $2.75 \mu$ of luciferin substrate (Promega) in dd- $\mathrm{H}_{2} \mathrm{O}$. Prepared sensor reactions ( $50 \mu \mathrm{l}$ per well) were quickly deposited in-fabric to be snap-frozen and then lyophilized for $4-8 \mathrm{~h}$ within the device. Activation of sensors was achieved by rehydration with a fluid splash of dd $-\mathrm{H}_{2} \mathrm{O}$ spiked with $3 \mu \mathrm{M}$ B. burgdorferi trigger RNA freshly made for the positive samples, while $0 \mu \mathrm{M}$ trigger RNA was used for controls. These wFDCF sensors were tested at $30^{\circ} \mathrm{C}$ and ambient humidity to simulate surface body temperature.

Preparation of optimized CRISPR-Cas12a-based wearable synthetic biology reactions. CRISPR-based sensor reactions for wFDCF testing in Fig. $3 b-e, h$ and Extended Data Figs. 4 and 6 were prepared using 100 nM Cas12a (New England Biolabs) and $100 \mathrm{nM}$ gRNA, $1 \times$ NEB buffer 2.1, $0.45 \mathrm{mM}$ dNTPs, $500 \mathrm{nM}$ of each RPA primer, $1 \times$ RPA liquid basic mix (TwistDx), $14 \mathrm{mM} \mathrm{MgCl}_{2}$ and $5 \mu \mathrm{M}$ FAM-Iowa Black FQ quenched ssDNA fluorescent reporter (Integrated DNA Technologies) in dd- $\mathrm{H}_{2} \mathrm{O}$. Prepared sensor reactions ( $50 \mu \mathrm{l}$ per well) were quickly deposited in-fabric to be snap-frozen and then lyophilized for $4-8 \mathrm{~h}$ within the device. Activation of sensors was achieved by rehydration with a fluid splash of dd $-\mathrm{H}_{2} \mathrm{O}$ spiked with $2.7 \mathrm{fM}$ or $100 \mathrm{fM}$ of $m e c A$, spa or ermA DNA trigger depending on the demonstration. In the sensing performed at $2.7 \mathrm{fM} \mathrm{mecA}$ trigger, the detection limit is 10,000 copies of DNA per $\mu$ l. These wFDCF sensors were tested at $30^{\circ} \mathrm{C}$ and ambient humidity to simulate surface body temperature. All of the CRISPR-based wFDCF sensor plots are the average of three independent wells. Each well contained three separate fiber optic sensors, for a total of nine fiber outputs presented per variable. Statistical significance values for specific time points were calculated using unpaired parametric Student's $t$-test (one-sided)

Preparation of optimized CRISPR-Cas13a-based wearable synthetic biology reactions. CRISPR-Cas13a-based sensor reactions for wFDCF testing (Extended Data Fig. 5) were prepared using $100 \mathrm{nM}$ Cas13a and $100 \mathrm{nM}$ gRNA, $1 \times$ NEB buffer 2.1, $0.45 \mathrm{mM}$ dNTP, $14 \mathrm{mM} \mathrm{MgCl}_{2}$ and $5 \mu \mathrm{M}$ FAM-Iowa Black FQ quenched RNA fluorescent reporter (Integrated DNA Technologies) in dd- $\mathrm{H}_{2} \mathrm{O}$. Prepared sensor reactions $(50 \mu \mathrm{l}$ per well) were quickly deposited in-fabric to be snap-frozen and then lyophilized for $4-8 \mathrm{~h}$ within the device. Activation of sensors was achieved by rehydration with a fluid splash of dd- $\mathrm{H}_{2} \mathrm{O}$ spiked with $20 \mathrm{nM}$ of MRSA RNA trigger.

Preparation of sample lysis-integrated wearable synthetic biology reactions. For wFDCF with integrated lysis reactions, an RNase-free Whatman filter paper disc $(8 \mathrm{~mm})$ was filled with concentrated stock solutions that would yield, upon a $50-\mu$ rehydration volume, $5 \mathrm{mM}$ Tris- $\mathrm{HCl}$ ( $\mathrm{pH} 7.5$ ), $1 \%$ Triton X-100, $1 \% \mathrm{NP}-40$, $0.2 \%$ CHAPS, $100 \mathrm{\mu g} \mathrm{ml}^{-1}$ lysozyme and $5 \%$ sucrose. This was freeze-dried for $4 \mathrm{~h}$ and inserted into the POF wFDCF device below the blackout layer and above a PVA time delay barrier that was sealed around the edges with Ecoflex elastomer to enable an efficient lysis incubation time. All layers containing the lyophilized RPA-Cas12a synthetic biology sensors below the lysis-PVA delay layers were identical to that used in the mecA RPA-Cas12a devices shown in Fig. 3b-e.

Garment-level integration of colorimetric synthetic biology sensors. After fabrication of a colorimetric synthetic biology wearable module, a bracelet 'garment' was achieved simply by gluing the module into an elastic band to be placed on the forearm of a mannequin (Supplementary Fig. 3c).

\section{Garment-level integration of fluorescence/luminescence synthetic biology} sensors. After fabrication of at least 12 fluorescence/luminescence synthetic biology wearable modules, a commercially available long-sleeve neoprene wetsuit-type jacket (EYCE Dive \& Sail) was modified to integrate an array of wFDCF sensors by sewing these modules in predefined high-splash-frequency regions (Figs. 2a and 4a and Extended Data Fig. 8f). Reaction modules were covered at the edges with a blackout fabric border with textile adhesive. POF bundles of these modules were sewn internally and directed to a single multi-bundle arrangement for interrogation via our portable spectrometer device (located in a back pocket within the jacket) The base neoprene fabric used for this jacket was of 3-mm thickness and treated with a superhydrophobic coating to prevent fluid absorption in places other than the reaction zones. The fabricated wFDCF jacket prototype was specified to fit a medium-sized male torso (36" chest by 31 " waist). In-garment sensors were tested on a mannequin at room temperature.

Construction and preparation of SARS-CoV-2 A-version diagnostic face mask. The SARS-CoV-2 in-mask diagnostic consists of the sensor assembly containing the lyophilized reactions which was then inserted into an N95-equivalent face mask (Fig. 4a for a schematic of the sensor; Fig. $4 \mathrm{~b}$ and Supplementary Fig. 14 for fully assembled face masks). First, capillary wicking material (porous Porex HRM (high-release media) fiber media (no. 36776, Porex Filtration Group), thickness $=0.5 \mathrm{~mm}$, density $=0.07 \mathrm{gcc}^{-1}$, porosity $=92 \%$ ) was laser-cut into a shape allowing for an elliptical region approximately $50 \times 25 \mathrm{~mm}^{2}$ that serves as the sample collection area, accumulating viral particles from a patient's respiration, vocalization and/or reflexive tussis. The laser-cut wicking material is then adhered to a white PET double-adhesive backing material (3M Microfluidic Diagnostic
Tape, no. $9965(3 \mathrm{M}))$. One end of the wicking material is adhered to a sterile sealed blister-pack containing nuclease-free water. The $\mu \mathrm{PAD}$ device is created by wax printing hydrophobic patterns onto Whatman Grade 1 chromatographic filter paper (Thermo Fisher Scientific) using a Xerox Phaser 8560 solid ink printer. The printed $\mu \mathrm{PAD}$ sheets were then wax reflowed by hot pressing for $15 \mathrm{~s}$ at $125^{\circ} \mathrm{C}$ using a Cricut EasyPress (Cricut), and then left untouched to cool at room temperature. After wax reflow, the reaction zones have an aperture diameter of $5 \mathrm{~mm}$, while the intervening PVA time delays have an aperture diameter of $3 \mathrm{~mm}$. The PVA time delays were placed onto the time-delay zones first, by pipetting $4 \mu \mathrm{l}$ of 10\%, 67,000 average molecular weight PVA (MilliporeSigma) per delay layer, and allowing it to dry at room temperature overnight. The lysis buffer, RT-RPA reaction and the Cas12a SHERLOCK reactions as described below were then added to the respective lysis zones.

The lysis reaction added to each sensor lysis zone was $15 \mu \mathrm{l}$ of $10 \mathrm{mM}$ Tris- $\mathrm{HCl}$ (pH 7.5), $1 \%$ Triton X-100, $1 \%$ NP-40, 0.2\% CHAPS, $100 \mu \mathrm{g} \mathrm{ml}^{-1}$ lysozyme and $5 \%$ sucrose. The RT-RPA reaction added to the isothermal amplification zone was $15 \mu$ l of a single lyophilized TwistAmp RPA pellet (TwistDx) that was rehydrated to $50 \mu \mathrm{l}$ using a rehydration reaction of $29.6 \mu \mathrm{l}$ of Twist Rehydration Buffer and $9.6 \mu \mathrm{l}$ of a primer mix (Supplementary Table 2; RT-RPA-F4, RT-RPA-R4 and RT-RPA-R3 primers in the mix are at a ratio of $10 \mu \mathrm{M} / 10 \mu \mathrm{M} / 20 \mu \mathrm{M})$. Roche Protector RNase Inhibitor, TAKARA PrimeScript Reverse Transcriptase and Ambion RNase H were all added at $1 \mu \mathrm{l}$ each. Nuclease-free water was added at $4.4 \mu \mathrm{l}$. Immediately before pipetting onto the reaction zone, $2.5 \mu \mathrm{l}$ of $280 \mathrm{mM} \mathrm{MgOAc}$ was added to the RT-RPA reaction and thoroughly mixed. For the Cas12a SHERLOCK reaction, $15 \mu \mathrm{l}$ of the following reaction was pipetted onto the SHERLOCK reaction zone: $12.3 \mu \mathrm{l}$ of nuclease-free water, $1.5 \mu \mathrm{l}$ of NEB Buffer $2.1,0.3 \mu \mathrm{l}$ of $0.5 \mathrm{M}$ DTT, $0.075 \mu \mathrm{l}$ of $100 \mu \mathrm{M}$ NEB EnGen Lba Cas12a and $0.26 \mu \mathrm{l}$ of $40 \mu \mathrm{M}$ coronavirus S-gene gRNA. Immediately before pipetting onto the reaction zone, 1 pmol of the 6-FAM/ TTATTATT/Biotin oligo (FB probe, from Integrated DNA Technologies) was added to the Cas12a reaction and thoroughly mixed. Sequences for all primers, RNA targets and the gRNA are presented in Supplementary Table 2.

All reactions were pipetted onto the reaction zones and the wax-printed sheet is then dipped into liquid nitrogen to freeze all of the embedded reactions, and then immediately wrapped in foil and placed on a lyophilizer. After lyophilization for $4-24 \mathrm{~h}$, the wax arrays are removed from the lyophilizer. Cutting of the arrays into individual $\mu \mathrm{PAD}$ strips can be performed before or after the lyophilization process. Each strip is folded using sterilized tweezers into an overlapping accordion arrangement (as shown in Fig. 4b), overlapping the reaction zones and time delays to form a $\mu \mathrm{PAD}$ device. The output end of the laser-cut Porex sample collection section was carefully inserted on top of the lysis zone, while the input end of a Milenia HybriDetect-1 Universal Lateral Flow Assay (TwistDx) was inserted on top of the last PVA time delay. The entire $\mu$ PAD section was carefully sandwiched and taped together to compress all of the layers. The entire blister-pack water reservoir-Porex sample collection area- $\mu$ PAD-LFA test strip is secured using the double-sided backing to the inside of an N95-equivalent mask, positioning the sample collection area in the region directly in front of the mouth and nose. The LFA test strip is routed to the outside of the mask through a small slit in the mask and the indicator has been oriented to hide the results from external viewing, to ensure patient confidentiality. To access the results, the test strip must be bent over to view the results (Supplementary Fig. 14g). Lastly, a button is affixed to the outside of the mask directly over the water blister reservoir. The button contains a small spike embedded in a compressible foam double-sided adhesive material. When pressed down, the button pierces the foil on the blister, allowing the nuclease-free water to flow through the same collection zone, the $\mu \mathrm{PAD}$ reaction zones and time delays, and finally into the LFA indicator strip. The modular design of the sensor components allows elements such as the water reservoir, $\mu$ PAD or LFA strip to be adjusted for different orientations or placement on the inside or outside of the mask. Only the sample collection pad module has strict orientation and positional requirements.

Bench-top testing of A-version SARS-CoV-2 diagnostic face-mask sensors. For Fig. 4d,f, each data point consisted of a face-mask sensor in which a defined amount of synthetic SARS-CoV-2 RNA fragment containing the specific gRNA-targeting region of the SARS-CoV-2 spike gene was generated by in vitro transcription using the HiScribe T7 Quick High Yield RNA Synthesis Kit (NEB) using synthetic DNA templates with a T7 promoter (Integrated DNA Technologies and Twist Bioscience). Corresponding homologous regions to the spike gene for the commonly circulating HCoV strains 229E, HKU1, NL63 and OC43 were determined by sequence homology alignment of the respective spike genes (Supplementary Fig. 14a) and the RNA targets were generated using the same method described above. All SARS-CoV-2 face-mask sensors were tested at room temperature at ambient humidity. After activation and LFA output formation $(\sim 20-30 \mathrm{~min})$, the LFA strips were digitized using the scanner function on a Ricoh MP C3504 on default contrast settings. This ensured equal brightness and contrast across all strips in comparison with photography. Each test (T) and control (C) output line from each strip was quantified in ImageJ from the 32-bit converted raw scanned images without any adjustments to brightness or contrast.

Fabrication of B-version face-mask sensors. The following optimizations to the A-version sensors were implemented, resulting in the improved B-version. 
Wax-printed $\mu \mathrm{PAD}$ templates were prepared as described above for the A-version sensors with the following changes (Supplementary Fig. 15a,b). To prevent failure from flow leakage between different layers of the folded $\mu \mathrm{PAD}$, unwaxed borders were rendered hydrophobic by drawing over the area with a Super PAP Pen (ThermoFisher) and allowed to air-dry for at least $1 \mathrm{~h}$. The sample collection pads for the B-version sensors were laser-cut from sheets of Porex high-release media no. 36776 with the dominant fiber direction along the long axis of the pad to allow faster flow of the hydration front. The pad geometry was adjusted to enhance water flow by moving the reservoir puncture point to the distal end of the water blister, increasing the pad area in contact with the water reservoir, and reducing the sample collection region. Approximately $2 \mathrm{~mm}$ of the outer border of the sample pad was rastered during laser-cutting to heat-seal the Porex material to the PET backing material, preventing delamination. Before assembly, approximately $1 \mathrm{~cm}$ of the backing material was peeled away and cut off from the end of the sample pad region that is to be in contact with the reservoir.

Before the addition of the reagents to the $\mu \mathrm{PAD}$, each reaction zone area was blocked with $5 \mathrm{ml}$ of $1 \% \mathrm{BSA}+0.02 \%$ Triton X-100 and allowed to air-dry for $12 \mathrm{~h}$ to prevent nonspecific adsorption of the biochemical reaction components to the filter paper matrix. PVA at a concentration of $18 \%(\mathrm{w} / \mathrm{v})$ at a volume of $\sim 5 \mu \mathrm{l}$ was applied to each time delay zone and allowed to air-dry for $24 \mathrm{~h}$. The lysis buffer for the B-version sensors was reformulated to $10 \mathrm{mM}$ Tris- $\mathrm{HCl}$ ( $\mathrm{pH} 7.5$ ), $5 \%$ sucrose, $0.02 \%$ NP-40 and 2\% CHAPS. The amount of nonionic surfactants in the lysis buffer was reduced to prevent observed degradation of the wax barrier, an observation we had made during design and testing of the A-version $\mu$ PADs. The CHAPS concentration was increased as it was not found to degrade the wax and this zwitterionic detergent has previously been shown to be effective in lysing coronavirus particles ${ }^{39}$. A volume of $10 \mu \mathrm{l}$ of this lysis buffer was added to the $\mu$ PAD lysis zone. The RT-RPA and Cas12a SHERLOCK reaction compositions, volumes and lyophilization parameters were unchanged. During final assembly of the B-version sensor, both the sample pad:: $\mu \mathrm{PAD}$ and the $\mu \mathrm{PAD}:: \mathrm{LFA}$ contact regions were fully sealed using precut sterile aluminium PCR foil seals (no. 60941-076, VWR) to improve contact transfer and prevent any fluidic short-circuiting that may occur from undesired droplet contact to the folded $\mu \mathrm{PAD}$ edges. To facilitate unimpeded sample flow, venting holes were introduced into the water-containing blister mold to prevent vacuum buildup inside the blister during flow. Three venting holes were punched into the blister surface using an 18-gauge needle and then sealed with a 6-mm adhesive disc of a single-sided rayon breathable hydrophobic porous film (no. 60941-086, VWR). This allows venting of vacuum while preventing leakage and contamination of the nuclease-free water. For all B-version face-mask-integrated sensors, the water reservoir module was positioned on the exterior of the mask to minimize unwanted contact pressure on the blister-pack during wearing of the mask. The sensor activation mechanism is the same as the A-version sensors. To integrate the sensors into the face masks, $1-\mathrm{cm}$ slits were cut into KN-95 masks through which the sensor ends were threaded and subsequently sealed using adhesive.

Breathing simulator apparatus assembly. Our face-mask sensor testing platform (Fig. 4h and Supplementary Fig. 16) consisted of four modules that performed the following functions: spontaneous breath generation, aerosol production, heating control, and physiologic airway and head simulation. For the breath generation, we employed the TestChest Lung Simulator (Organis), a highly accurate artificial lung that uses an actuated dual bellows design to replicate lung mechanics such as lung vial capacity and tidal volume. The TestChest was connected through ventilator tubing to all other downstream modules for simulated spontaneous breathing. Directly downstream of the TestChest, we placed an in-line Aerogen Solo nebulizer (Aerogen). The Aerogen Solo is a medical-grade vibrating-mesh nebulizer for the administration of lung inhalation therapeutics. Previous studies have demonstrated that the nebulizer generates aerosol droplets that are similar in diameter to those that occur naturally from human lung emissions ${ }^{50}$. Furthermore, previous work has used the Aerogen system to deliver therapeutic RNA in an animal model ${ }^{51}$, showing that it can be used to produce transmissible RNA-laden aerosols. The tubing is next wrapped in a temperature-regulated heat pad (Zoo Med Laboratories) that maintains the output temperature at $35^{\circ} \mathrm{C}$. The tubing is connected to a lung input tube in a high-fidelity airway manikin (7-SIGMA Simulation Systems) that faithfully replicates pulmonary and nasopharyngeal structures as well as head movement ranges. The other simulated lung and the simulated esophagus are clamped shut to direct breath output only through the oral cavity.

On-simulator testing of face-mask-integrated B-version sensors. For all simulator-based testing, a SARS-CoV-2 B-version sensor-containing face mask was fitted onto the 7-SIGMA airway manikin and the TestChest was set to the 'Normal Stable' setting, which generates a spontaneous breathing rate of 12 breaths per minute. The entire breathing simulator assembly was then checked for leaks. Temperature regulation was set to maintain an outflow temperature of $35^{\circ} \mathrm{C}$. A 5 -ml solution of SARS-CoV-2 F5R11 vRNA IVT target was then pipetted into the Aerogen Solo reservoir and the controller unit activated. The simulated breath was allowed to collect in the face mask and sensor for a period of $30 \mathrm{~min}$, then the sensor was activated on the manikin for processing while maintaining the breathing and heating. The LFA outputs for all sensors were scanned using a Ricoh MP C3504 printer system using default settings.
The total amount of aerosolized vRNA collected after $30 \mathrm{~min}$ on each mask sensor for a given concentration of vRNA IVT target solution was estimated by RT-qPCR analysis of a 6-mm filter paper disc affixed to the sample pad area. After the $30 \mathrm{~min}$ of the breathing simulation, the disc was removed and frozen immediately in nuclease-free microcentrifuge tubes at $-80^{\circ} \mathrm{C}$ for later analysis. Replicate disc collections were then repeated using the same procedure. For analysis, the discs were thawed and resuspended in $100 \mu \mathrm{l}$ of nuclease-free water supplemented with Protector RNase Inhibitor (Roche). RNA was extracted by repeated vortexing for 20-s burst intervals with resting on ice. This extracted sample was used as template in RT-qPCR reactions to obtain the total accumulated target RNA copy number on the 6-mm sampling disc. The mean collection values (in copies per $\mathrm{mm}^{2}$ ) are then multiplied by the exposed surface area of the sample collection pad $\left(2,513 \mathrm{~mm}^{2}\right)$ to estimate the total aerosolized vRNA target collected on the sensor. For a stock solution of $16.7 \mathrm{fM}$ vRNA IVT target, the estimated total collected copies per sensor is $2.3 \times 10^{6}$ copies. For a stock solution of $1.67 \mathrm{pM}$ vRNA IVT target, the estimated total collected copies per sensor is $5 \times 10^{7}$ copies. These values are reported in Fig. 4i,j. The scatter plots for each target concentration show the $\mathrm{T} / \mathrm{C}$ ratio from five independently fabricated and measured sensors.

Sensor and reporter sequences. Supplementary Tables 2 and 3 contain the DNA and RNA sequences of sensors and reporters used in this study. The plasmid construct used for the Zika 27B toehold sensor has been previously described elsewhere ${ }^{52}$. The Lyme disease and HIV toehold sensors with an nLuc output were cloned into the pBW121 plasmid backbone (Addgene plasmid no. 68779). All other plasmid constructs utilized the pJL1 backbone that has been previously described ${ }^{12,14}$. The F30 dimeric Broccoli fluorescent aptamer was subcloned into pJL1 from pET28c-F30-2xd-Broccoli, which was a gift from Samie Jaffrey (Addgene plasmid no. 66843; http://n2t.net/addgene:66843; RRID: Addgene_66843). The sequence for the pJL1-sfGFP plasmid can be found on Addgene (plasmid no. 69496).

Reporting Summary. Further information on research design is available in the Nature Research Reporting Summary linked to this article.

\section{Data availability}

All data needed to evaluate the conclusions in the paper can be found in the paper and the Supplementary materials. Correspondence and requests for materials should be addressed to J.J.C.

\section{Code availability}

The custom code developed for this work is provided in the Supplementary materials.

\section{References}

50. Fennelly, K. P. Particle sizes of infectious aerosols: implications for infection control. Lancet Respir. Med. 8, 914-924 (2020).

51. Patel, A. K. et al. Inhaled nanoformulated mRNA polyplexes for protein production in lung epithelium. Adv. Mater. 31, e1805116 (2019).

52. Pardee, K. et al. Rapid, low-cost detection of Zika virus using programmable biomolecular components. Cell 165, 1255-1266 (2016).

\section{Acknowledgements}

We thank Samsara S.r.l--Dreamlux for aid in the custom fabrication of the wearable fabrics incorporating flexible fiber optics. Our gratitude to G. Vis from SimVS for providing access and support for the TestChest Lung Simulator. In addition, we thank T. E. Reihsen from 7-SIGMA Simulation Systems (7S3) for providing the high-fidelity airway trainer. We are also indebted to X. Tan for consultations on clinical considerations for the SARS-CoV-2 face-mask design. We also acknowledge T. Ferrante for support in the design and fabrication of the optical wearable acquisition device. Materials for the face mask prototype were kindly contributed by $3 \mathrm{M}$ through $\mathrm{A}$. Brick. We further thank J. Gootenberg, O. Abudayyeh and the Zhang Lab for providing us with Cas13a enzyme. The nanoluciferase plasmid was kindly donated by D. Thompson from the Church Lab. The pET28c-F30-2xd-Broccoli plasmid was a gift from S. Jaffrey (Addgene plasmid no. 66843). We also thank J. W. Lee for aid in general toehold sensor design, as well as A. Dy and M. Takahashi for discussions and advice relating to the implementation of cell-free synthetic biology circuits. This work was supported by the Defense Threat Reduction Agency grant no. HDTRA1-14-1-0006, the Paul G. Allen Frontiers Group, the Ragon Institute of MGH, MIT and Harvard award no. 234640, the Patrick J. McGovern Foundation, the Wyss Institute for Biologically Inspired Engineering, Harvard University (J.J.C., P.Q.N., L.R.S., N.M.A.-M., H.P., N.M.D.) and by Johnson \& Johnson through the J\&J Lab Coat of the Future QuickFire Challenge award 2018. L.R.S. was also supported by CONACyT grant no. 342369/408970, and N.M.A.-M. was supported by an MIT-TATA Center fellowship, no. 2748460.

\section{Author contributions}

P.Q.N. and L.R.S. designed and constructed devices, planned and performed experiments, analyzed the data and wrote the manuscript. N.M.D., N.M.A.-M. and H.P. 
designed and performed experiments and analyzed the data. A.H. and T.G. performed experiments and edited the manuscript. S.S., T.G. and E.M.Z. assisted with aspects of design and construction of sensors or devices. R.L. optimized parts of the freeze-dried reactions. G.L., H.M.S. and J.B.N. contributed to concept development of the face mask. J.J.C. directed overall research and edited the manuscript.

\section{Competing interests}

The authors have submitted provisional patent applications based on the technology described in this manuscript. J.J.C. is a co-founder and board member of Sherlock Biosciences.

\section{Additional information}

Extended data is available for this paper at https://doi.org/10.1038/s41587-02100950-3.

Supplementary information The online version contains supplementary material available at https://doi.org/10.1038/s41587-021-00950-3.

Correspondence and requests for materials should be addressed to J.J.C.

Peer review information Nature Biotechnology thanks the anonymous reviewers for their contribution to the peer review of this work.

Reprints and permissions information is available at www.nature.com/reprints. 
a

Pre-lyophilization PURE reaction volumes

\section{$1 \cdot X$}

$1.25 \cdot X$
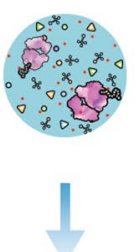<smiles>C1[C]2C[C]1C2</smiles>

\section{$1.5 \cdot X$}

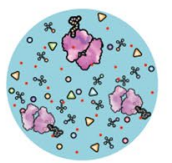

$2 \cdot X$

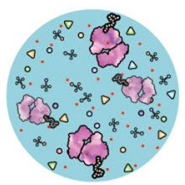

2.5•X

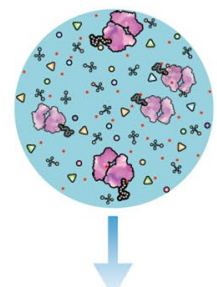

Lyophilization
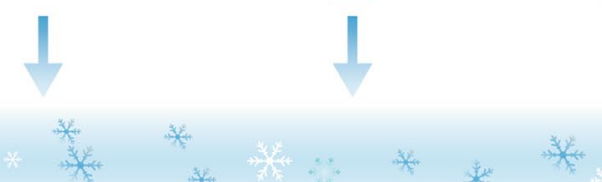
$\rightarrow(x)$
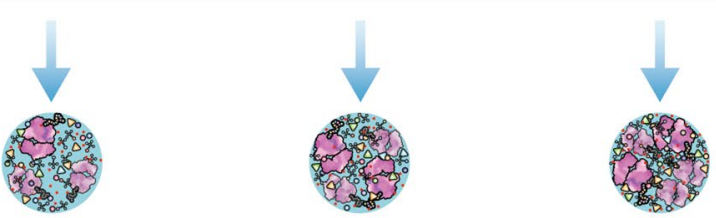

b

$X$ volume final

rehydration

to concentrate
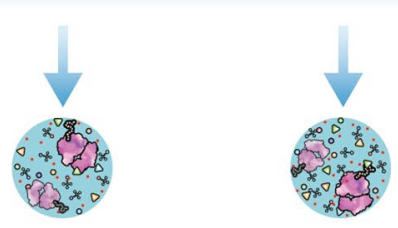

C
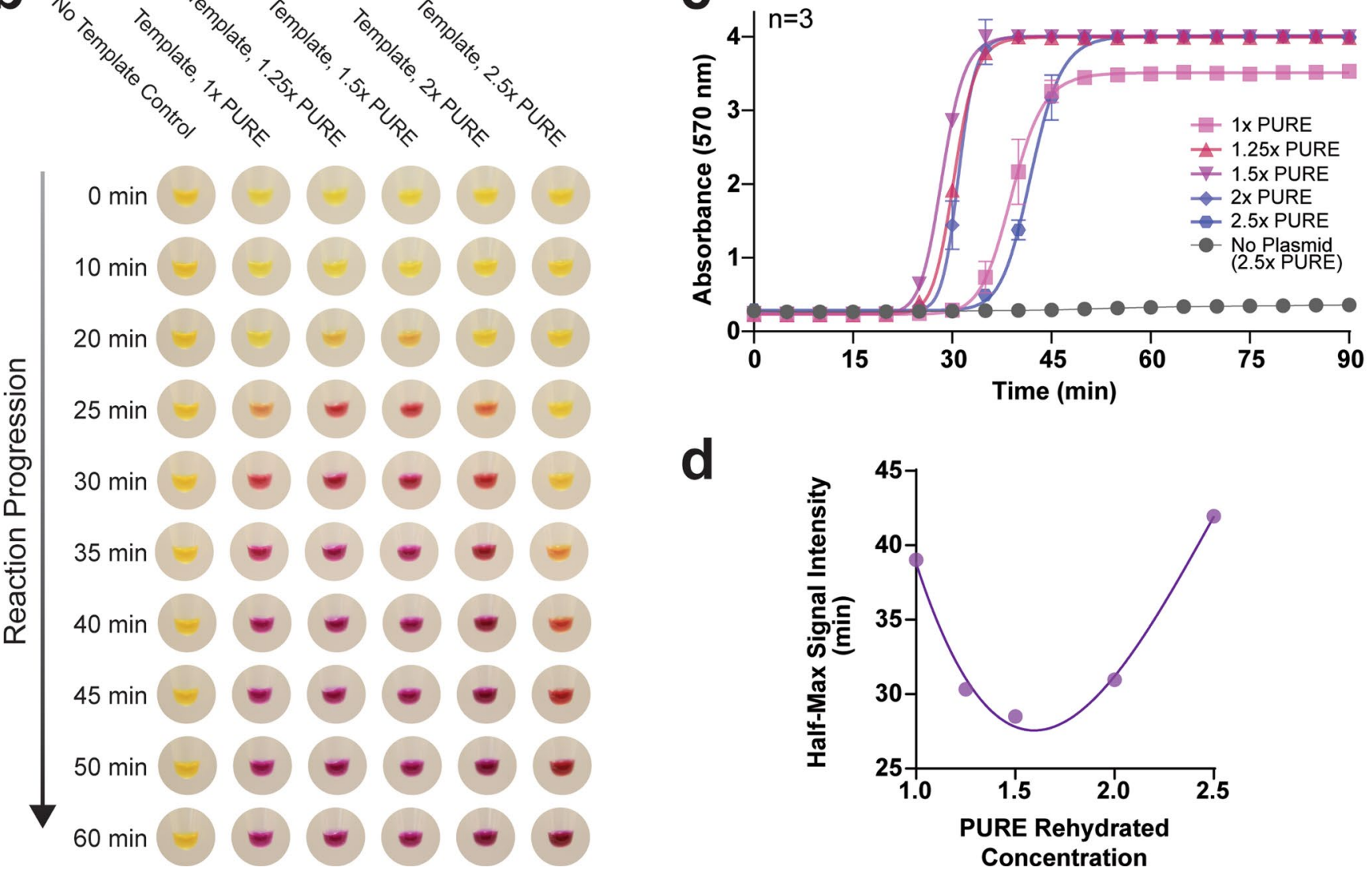

Extended Data Fig. 1 | Concentrating PURE cell-free reactions increases reaction kinetics. a, Schematic of reaction concentration through the Iyophilization of PURExpress reactions at varying volumes followed by rehydration at a set volume. Using this method, synthetic biology reactions can be concentrated to enhance kinetics through molecular crowding effects or greater density of cell-free components per volume. $\mathbf{b}$, Representative images of PURE reactions with a LacZ output over one hour, at various concentrations. c, Quantified PURExpress reactions with a LacZ output in triplicate; the error bars denote standard deviation. Plots here show mean \pm s.d. values for $n=3$ independent experiments of rehydrated PURExpress reactions in a microplate assay. $\mathbf{d}$, The half-maximal values from curve fitting the data shown in panel $\mathrm{c}$ indicate that the $1.5 \mathrm{x}$ concentrated PURE reaction accelerates the signal output by more than 10 minutes. Propagated error bars here are smaller than the data points. 
a

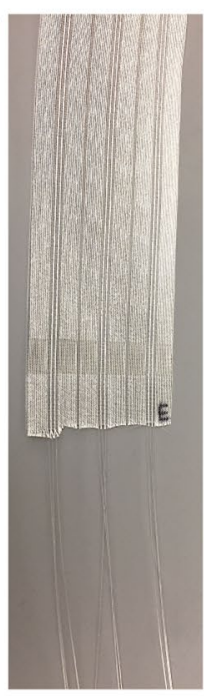

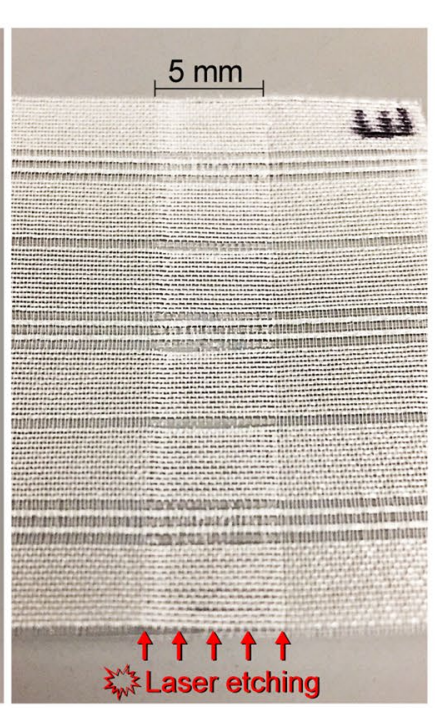

b

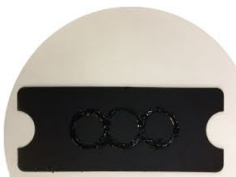

Bottom

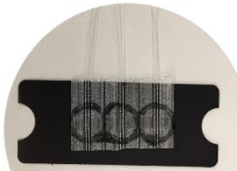

Excitation

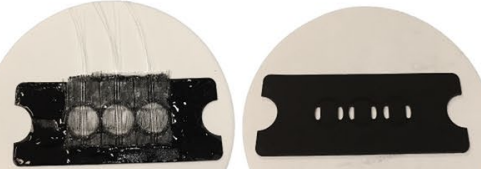

Top
C

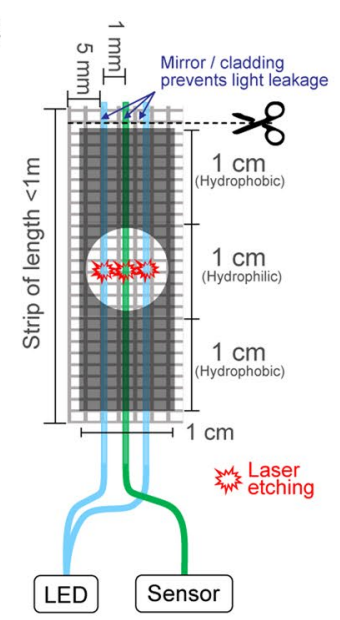

d

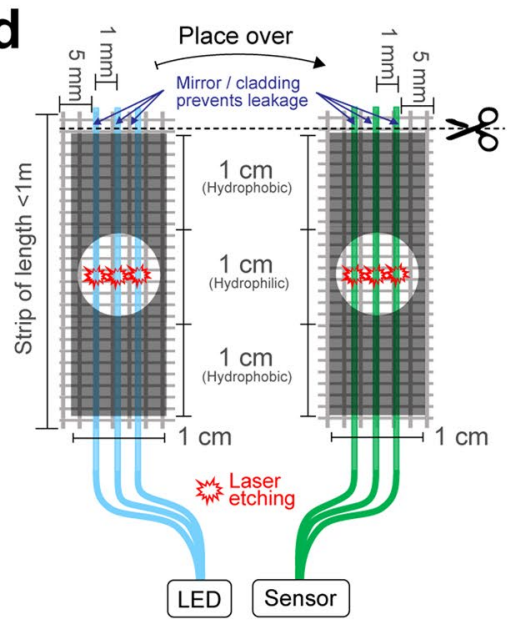

e

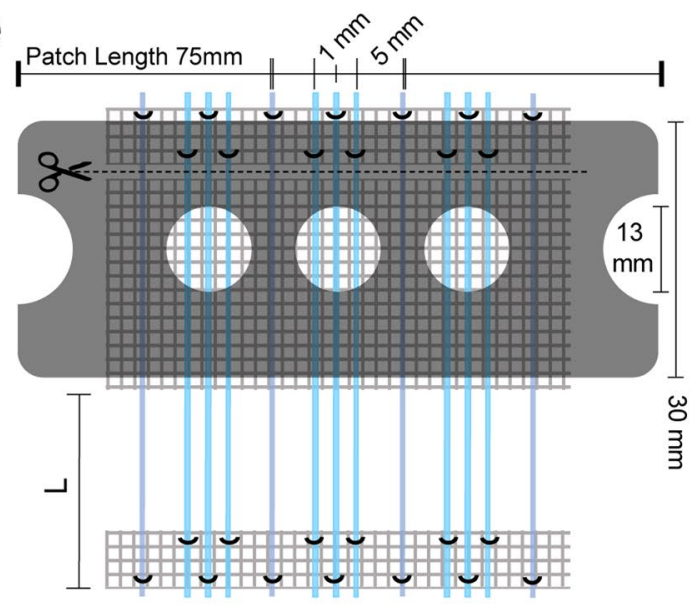

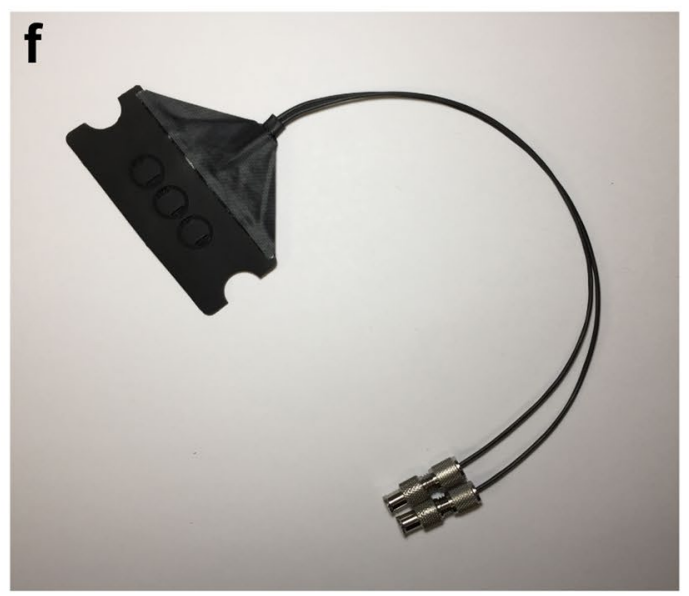

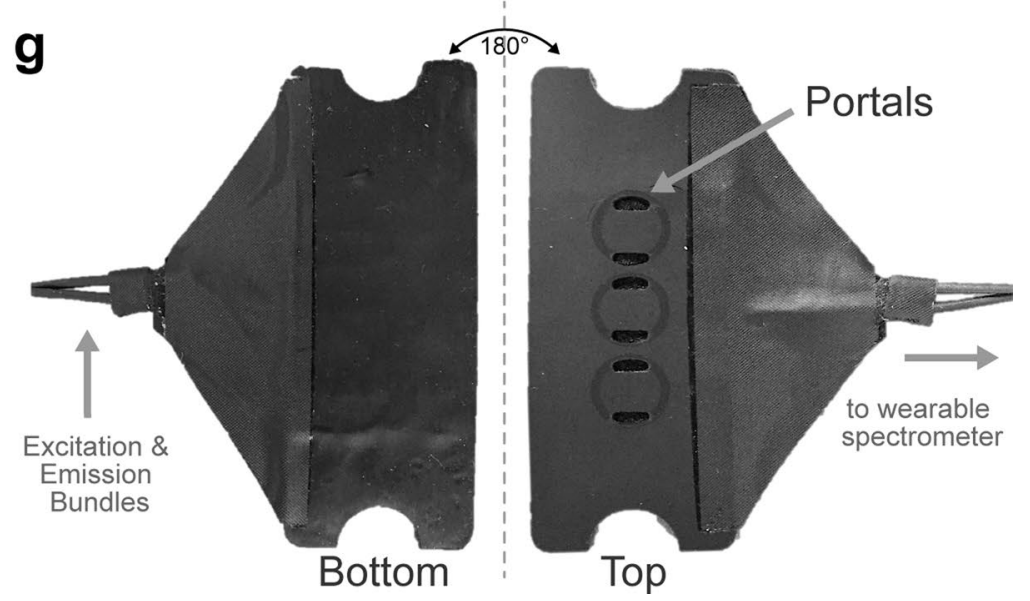

Extended Data Fig. 2 | See next page for caption. 
Extended Data Fig. 2 | Fabrication of fiber optic textile-based wFDCF sensor patch. a, A cut strip of hydrophilic POF fabric was laser-etched (5 mm) to ablate the POF outer cladding in the POFs sections closest to the reaction zone. $\mathbf{b}$, Examples of prepared wFDCF fabric-elastomer layers and final assembly into a three-well sensor for garment integration. The POFs in these devices were covered with black heat shrink tubing ( $6 \mathrm{~mm}$ ). Top elastomer cover features two $5.19 \times 1.85 \mathrm{~mm}$ curved sample ports instead of three as in the colorimetric prototypes to reduce direct light leakage on top of the POFs that may cause background light detection. c, Schematic of a POF-fabric-elastomer strip for sensing in a single textile layer including two excitation fibers on the sides of an emission fiber. $\mathbf{d}$, Schematic of a double POF-fabric-elastomer strip for sensing with dedicated excitation and emission layers. This design was the one selected for further experiments due to higher hydrophilic fiber content and capacity to immobilize fluid for lyophilization. e, Schematic of a single excitation or emission POF-fabric-elastomer layer overlaid on an applied elastomer pattern for creating the impermeable reaction wells. $\mathbf{f}, \mathrm{A}$ finalized three-well sensor WFDCF device with heat shrunk POF covers and Luer connectors for interfacing with a portable spectrometer device. $\mathbf{g}$, Top and bottom views of a final three-well sensor wFDCF device. The blackout fabric can be seen through the sample wicking ports and serve to prevent environmental light penetration into reaction wells. 

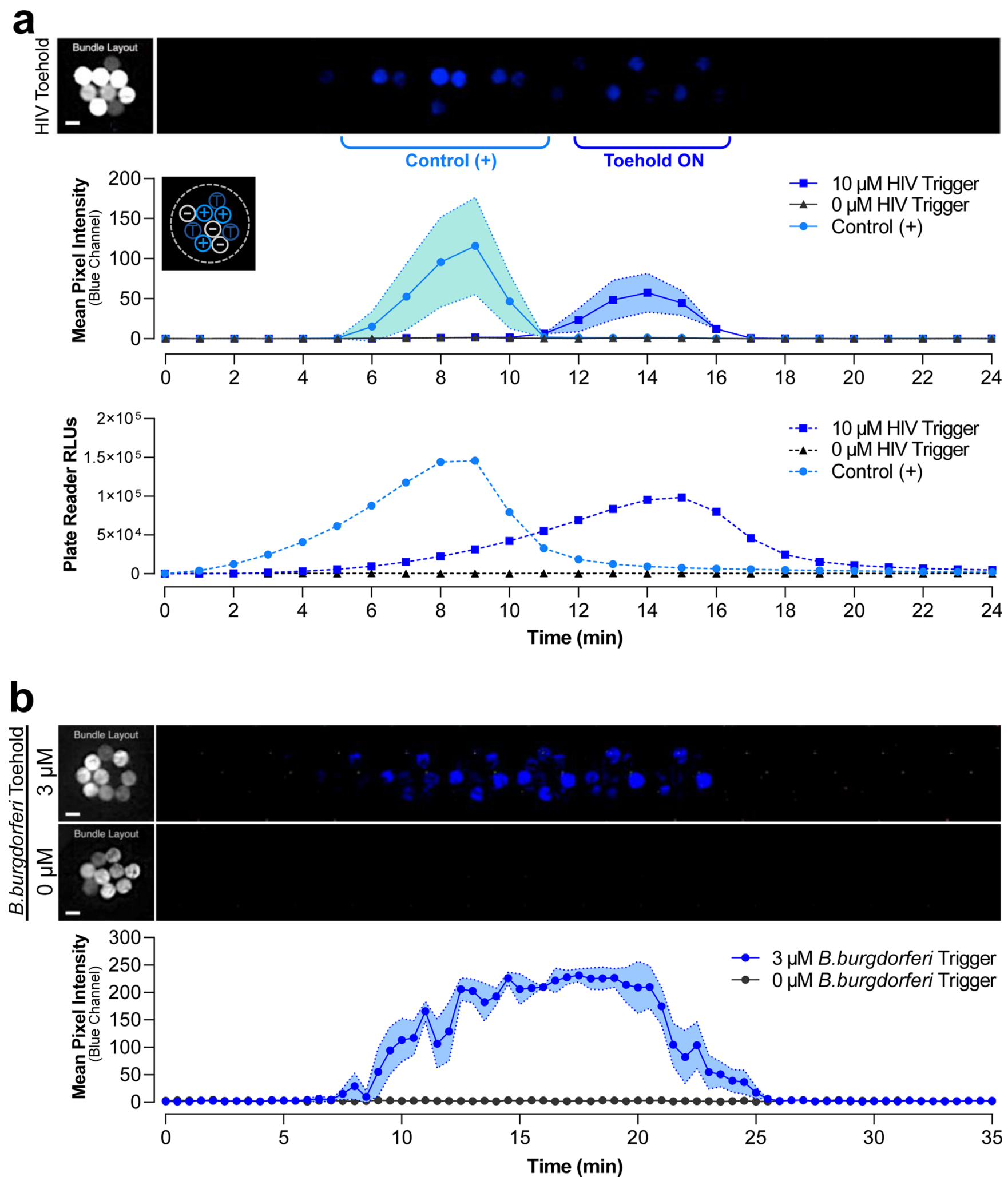

Extended Data Fig. 3 | See next page for caption. 
Extended Data Fig. 3 | Luminescent wFDCF toehold switches for B. burgdorferi and HIV detection. a, Dynamic response of a wFDCF HIV RNA toehold switch sensor with luminescence output in comparison to constitutive $P_{T 7}:$ :nLuc expression as a positive control ( + ). Activation of sensors was achieved by rehydration with a fluid splash of dd- $\mathrm{H}_{2} \mathrm{O}$ spiked with $10 \mu \mathrm{M} \mathrm{HIV}$ trigger RNA freshly made for the positive samples, while $0 \mu \mathrm{M}$ HIV trigger RNA was used for controls. The constitutive $\mathrm{P}_{\mathrm{T} 7}:$ :nLuc positive control reaction shown was also prepared similarly but substituting the toehold switch in the plasmid with a T7 promoter. Results (top) are compared to the same reactions run in a 384-well plate and analyzed using a plate reader in luminescence mode (bottom read). Inset shows identity of each individual fiber in the bundle, where T=HIV toehold with trigger, $(-)=$ HIV toehold without trigger, and $(+)$ is the $\mathrm{P}_{\mathrm{T} 7}:$ : $\mathrm{LLuc}$ control. Activation of constitutive reaction peaked at $\sim 8$ minutes, whereas toehold with $10 \mu \mathrm{M}$ trigger produced its peak signal at $\sim 15$ minutes. Both the wFDCF device tests and the plate reader profiles appeared to be temporally aligned and exhibit analogous signal amplitude differences among reactions. b. Dynamic response of a wFDCF Lyme disease RNA toehold switch sensor with luminescence output. Activation of sensors was achieved by rehydration with a fluid splash of dd- $\mathrm{H}_{2} \mathrm{O}$ spiked with $3 \mu \mathrm{M}$ B. burgdorferi trigger RNA freshly made for the positive samples, while $0 \mu \mathrm{M}$ trigger RNA was used for controls. All sensors shown were activated by rehydration and incubated at a temperature of $30^{\circ} \mathrm{C}$ and a relative humidity of $35 \%$. The plots for the wFDCF results (a, b-top panel) show integrated mean pixel intensity (dark lines) \pm s.d. (shaded regions) for three independent experiments. Scale bars in brightfield images are $250 \mu \mathrm{m}$. The luminescence trace plot (b-bottom panel) presented as reference are singlicates for each condition. 


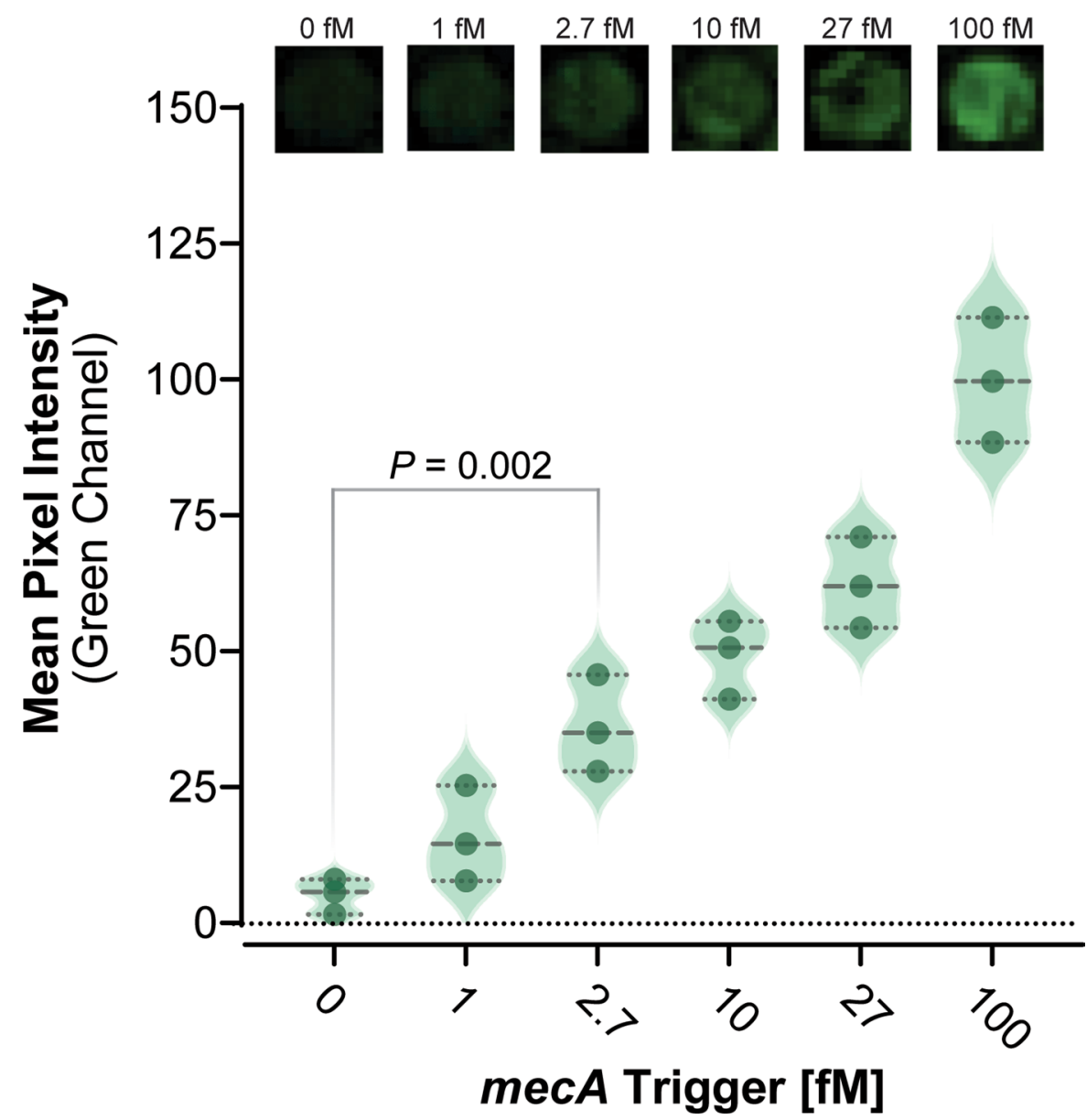

Extended Data Fig. 4 | Limit of detection of wFDCF CRISPR-Cas12a based sensor activated in-fabric. Our wFDCF mecA CRISPR-based sensor was exposed to various dsDNA trigger concentrations containing 0-100 fM mecA trigger, to assess in-fabric reaction fluorescence at $t=90$ min after fluid entry as compared to controls with a scrambled trigger. Increasing concentrations of trigger lead to an increase in fluorescence signal at the evaluation timepoint as denoted by the recorded mean pixel intensity from POF regions $(n=3)$. A statistically significant difference between the negative control and trigger presence was observed at 90 min only for concentrations equal and above that of $2.7 \mathrm{fM}$ of trigger $(P=0.002)$, which can be considered the limit of detection for this specific trigger, device configuration and evaluation timepoint. Violin plot shows median and quartiles as dotted lines and data points in dark green. Statistical differences were determined using unpaired one-tailed Student's t-test. 

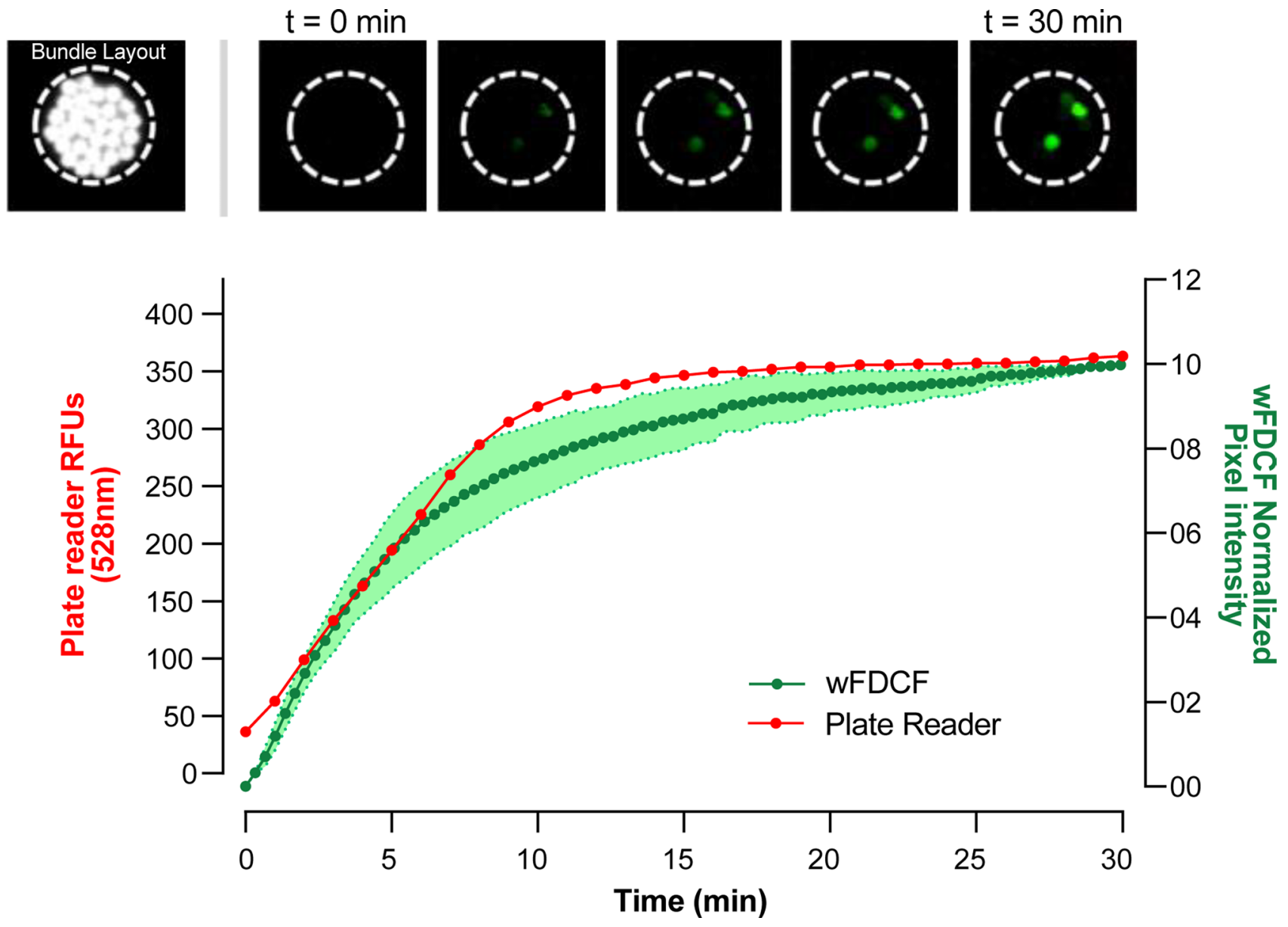

Extended Data Fig. 5 | Direct RNA detection in a wFDCF CRISPR-Cas13a based sensor. A CRISPR-Cas13a based MRSA SHERLOCK RNA sensor was prepared and freeze-dried over a wearable textile device for testing. All reactions contained RNaseAlert substrate, a quenched fluorophore probe that is cleaved by activated Cas13a (Integrated DNA Technologies, Coralville, IA). The wearable sensor was activated with a fluid splash of dd- $\mathrm{H}_{2} \mathrm{O}$ containing $20 \mathrm{nM}$ mecA RNA transcript trigger, while the plate samples were rehydrated with the same trigger concentrations to the originally deposited reaction volume $(4 \mu \mathrm{L})$. Reactions in the wFDCF were monitored at $30^{\circ} \mathrm{C}$ for 30 minutes using the wearable optical device and the reference control in a BioTek NEO HTS plate reader (BioTek Instruments, Inc., Winooski, VT) in fluorescence mode (Ex. $470 \mathrm{~nm} / \mathrm{Em}$. $528 \mathrm{~nm}$ ). Normalized pixel intensity in the wearable device is shown as mean (green dark line) \pm s.d. (green light region) of $n=3$ independent experiments and is comparable in dynamics to the results of the kinetic run conducted in the plate reader (red line) shown as a singlicate reference. 

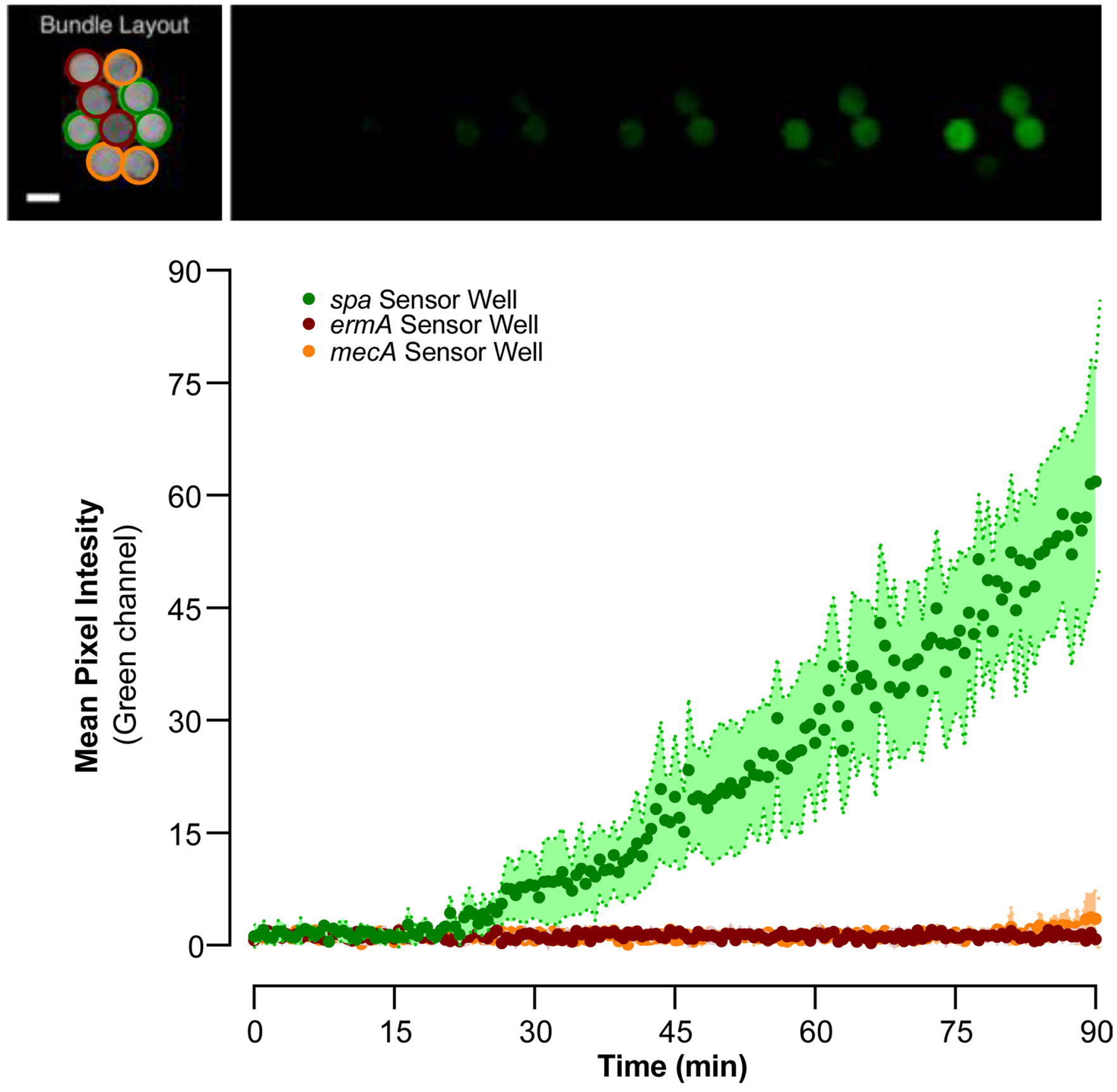

Extended Data Fig. 6 | Antibiotic resistance Cas12a sensors for spa, ermA and mecA genes using in-wearable wFDCF sensors demonstrate orthogonal specificity. Only reaction chambers with a Cas12a sensor targeting the $S$. aureus virulence factor-encoding spa-gene (colored in green) generates a detectable signal within $30 \mathrm{~min}$. The plot shows mean (dark points) \pm s.d. (light colored regions) for $\mathrm{n}=3$ individually fabricated and tested wFDCF reaction chambers for each sensor. The scale bar for the brightfield image of the fiber optic bundle is $250 \mu \mathrm{m}$. 


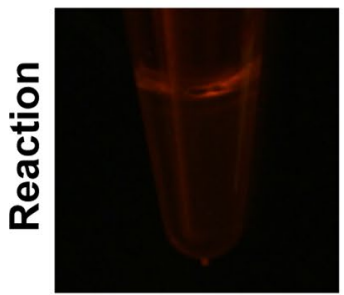

No Plasmid

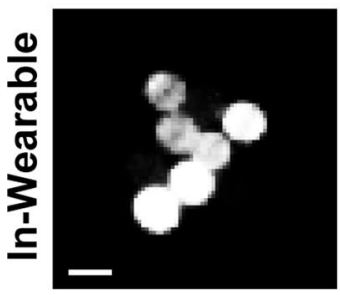

1. Brightfield

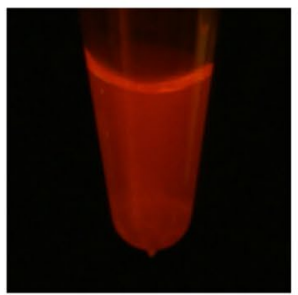

eforRed

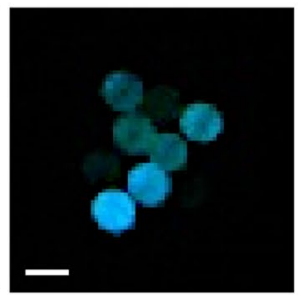

2. Dry

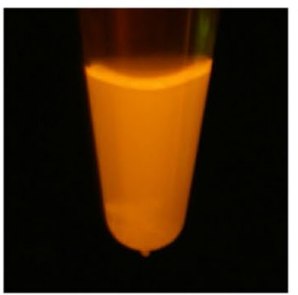

dTomato

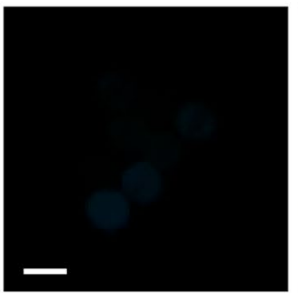

3. No Plasmid

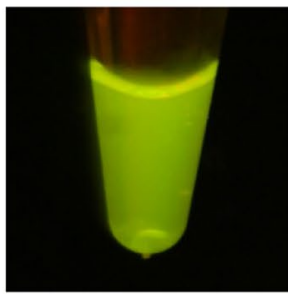

sfGFP

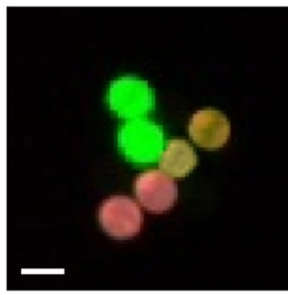

4. Multiplexed

Different

Fluorescent

Protein

Outputs

Extended Data Fig. 7 | Sensor multiplexing using different fluorescent proteins can be detected in a single wFDCF device. Top row, cell-free reactions in tubes demonstrating different fluorescent protein outputs generated post-rehydration from lyophilized FDCF PURE reactions after $30 \mathrm{~min}$ at $30^{\circ} \mathrm{C}$. All tubes were photographed with illumination using an Invitrogen Safe Imager 2.0 G6600 Blue Light Transilluminator (Carlsbad, CA). Bottom row, sensor images of WFDCF fiber bundle ends in (1) brightfield (intense light is placed over the laser-ablated POF sensor regions to spatially locate each fiber), (2) image when the sensor is dry, (3) image when wFDCF reaction is hydrated but without plasmid $\left(30\right.$ min incubation at $\left.30^{\circ} \mathrm{C}\right)$, and $(4)$ image when wFDCF reaction is hydrated with FP plasmids $\left(30 \mathrm{~min}\right.$ incubation at $30^{\circ} \mathrm{C}$ ). These images of the fiber optics are representative of experiments performed independently three times with similar results each time. Scale bars are $250 \mu \mathrm{m}$. 
a

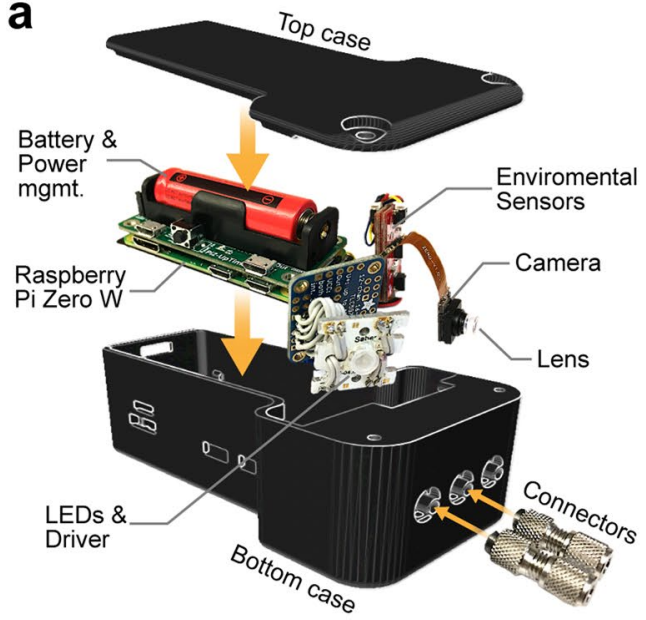

b

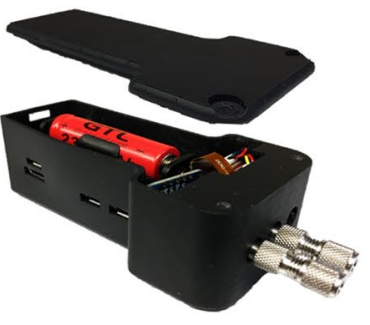

C

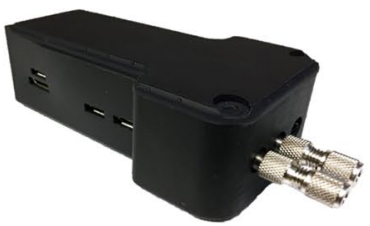

f

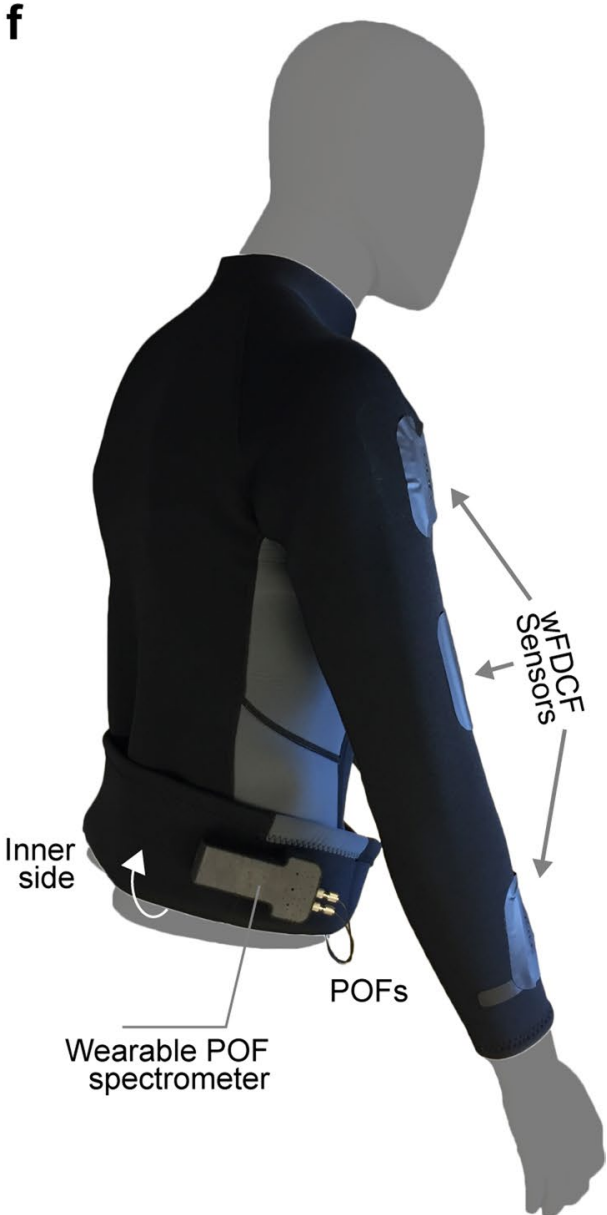

d

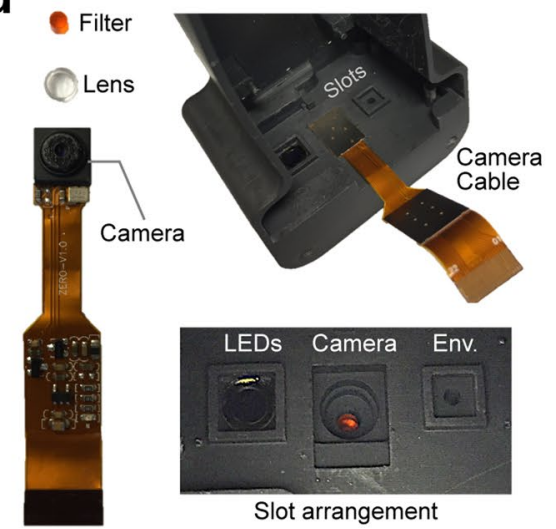

e

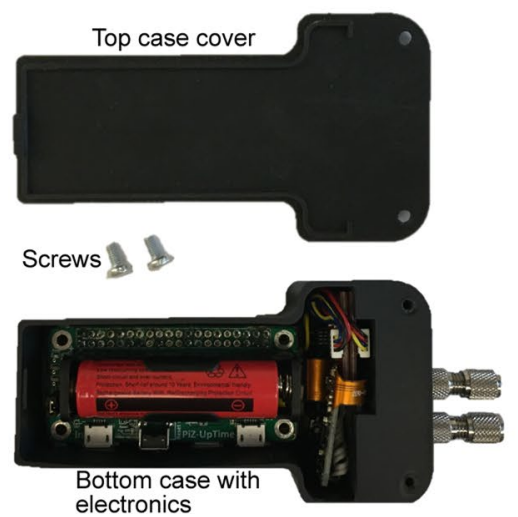

Extended Data Fig. 8 | Fabrication of wearable microcontroller system with LED illumination and spectrometric capabilities. a, Exploded isometric view of wearable POF spectrometer components with case and electronics. The device electronics are based on a Raspberry Pi Zero W Version 1.3 (Raspberry Pi Foundation, Cambridge, UK), assembled with a PiZ-UpTime battery power board (Alchemy Power Inc., Santa Clara, CA), an environmental sensing module, an LED illumination module, and a flexible camera for imaging. b. Photograph of an open assembled device. c, Photograph of a fully assembled device ready for imaging. d, Details of camera used in the device as well as the amber fluorescence emission filter and lens for magnification. Slots at the front of the bottom case fit the camera end, the LED arrangement and a vent for the environmental sensors. e, Top view of an assembled device to provide detail of compact electronics arrangement. f, Arrangement of wearable POF spectrometer with wireless connectivity in-garment for wFDCF reaction testing. 


\section{Reporting Summary}

Nature Research wishes to improve the reproducibility of the work that we publish. This form provides structure for consistency and transparency in reporting. For further information on Nature Research policies, see our Editorial Policies and the Editorial Policy Checklist.

\section{Statistics}

For all statistical analyses, confirm that the following items are present in the figure legend, table legend, main text, or Methods section. $\mathrm{n} / \mathrm{a}$ | Confirmed

$\bigotimes$ The exact sample size $(n)$ for each experimental group/condition, given as a discrete number and unit of measurement

Х A statement on whether measurements were taken from distinct samples or whether the same sample was measured repeatedly

The statistical test(s) used AND whether they are one- or two-sided

Only common tests should be described solely by name; describe more complex techniques in the Methods section.

Х $\square$ A description of all covariates tested

Х $\square$ A description of any assumptions or corrections, such as tests of normality and adjustment for multiple comparisons

$\square$ A full description of the statistical parameters including central tendency (e.g. means) or other basic estimates (e.g. regression coefficient)

$\triangle$ AND variation (e.g. standard deviation) or associated estimates of uncertainty (e.g. confidence intervals)

$\bigotimes \square \begin{aligned} & \text { For null hypothesis testing, the test statistic (e.g. } F, t, r \text { ) with confidence intervals, effect sizes, degrees of freedom and } P \text { value noted } \\ & \text { Give } P \text { values as exact values whenever suitable. }\end{aligned}$

Х $\square$ For Bayesian analysis, information on the choice of priors and Markov chain Monte Carlo settings

Х $\square$ For hierarchical and complex designs, identification of the appropriate level for tests and full reporting of outcomes

Х $\square$ Estimates of effect sizes (e.g. Cohen's $d$, Pearson's $r$ ), indicating how they were calculated

Our web collection on statistics for biologists contains articles on many of the points above.

\section{Software and code}

Policy information about availability of computer code

Data collection Two python-based executables for the Raspberry Pi Zero W were created, one for continuous stand-alone polymeric optic fiber signal acquisition, and another one for communication with blynk.io-based mobile application (Blynk Inc., New York, NY). The stand-alone sensing file "RPi_W_Python_CFFDWSB_V-1-0-7_A_Sens.py", and blynk.io-based mobile application code

"RPi_W_Python_CFFDWSB_V-1-0-7_B_Blynk.py" are included in the supplemental information and utilize only newly developed code and the following open-source (MIT licensed) modules: matplotlib 3.1.2, openCV 4.1.2, Python-based RaspberryPi camera 1.3.0 and GPIO 0.1.0 modules (Raspberry Pi Foundation, Cambridge, UK), as well as python-based TLC59711 1.0.0 AS7262 1.0.0, CCS811 1.0.0, BME280 1.0.0 control's modules (Adafruit Industries ${ }^{\oplus}$, New York, NY) and BlynkLib 0.2.5 from blynk.io (Blynk Inc., New York, NY).

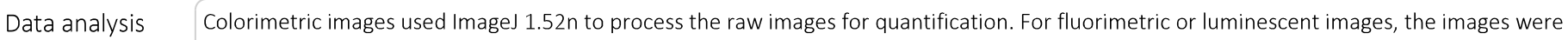
processed by custom Python code. For LFA band intensity analysis, Image $1.52 \mathrm{n}$ was used.

For manuscripts utilizing custom algorithms or software that are central to the research but not yet described in published literature, software must be made available to editors and

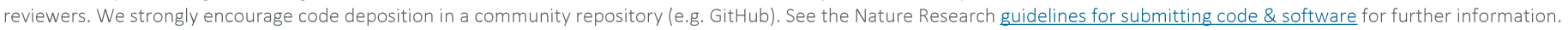


Policy information about availability of data

All manuscripts must include a data availability statement. This statement should provide the following information, where applicable:

- Accession codes, unique identifiers, or web links for publicly available datasets

- A list of figures that have associated raw data

- A description of any restrictions on data availability

All data needed to evaluate the conclusions in the paper can be found in the paper and the Supplementary Materials. Correspondence and requests for materials should be addressed to J.J.C.

\section{Field-specific reporting}

Please select the one below that is the best fit for your research. If you are not sure, read the appropriate sections before making your selection.

$\bigotimes$ Life sciences $\quad \square$ Behavioural \& social sciences $\quad \square$ Ecological, evolutionary \& environmental sciences

For a reference copy of the document with all sections, see nature.com/documents/nr-reporting-summary-flat.pdf

\section{Life sciences study design}

\section{All studies must disclose on these points even when the disclosure is negative.}

Sample size Sample sizes for the colorimetric wearables were selected at $n=3$ different sensors to provide a sufficient sampling of the performance of the designed devices and accounting for variations in lyophilization, rehydration, and reaction performance. For the fluorimetric wearable devices, each individual fiber optic bundle was treated as a data point, with 3 different reaction wells each with 3 POFs. For the SARS-CoV-2 face mask sensors, testing was performed on $n=4$ sensors for each variable on the benchtop or $n=5$ for each variable for on the breathing simulator apparatus.

Data exclusions For the fluorescent and luminescent data, for each array of sensors ( 3 wells, 9 total optical fibers), the total average pixel intensity was calculated. Any fibers below 1 SD of the mean were excluded from final analysis. The hand-assembled SARS-CoV-2 face mask sensors were carefully inspected for fabrication errors (misaligned uPADs, crushed sensors during lyophilization and poorly assembled sensors) and these were excluded from the experiment.

Replication Strict optimized procedures were taken to ensure lyophilization of the wFDCF reactions occurred similarly for all of the devices tested. All hydrated reactions were performed in temperature-controlled environments simulating human surface skin temperatures at ambient humidity to replicate field conditions. For the SARS-CoV-2 sensors, the microclimate of the mask was measured to maintain a temperature of $35 \mathrm{C}$ and relative humidity of $100 \%$ to simulate the microclimate inside of a human-worn facemask.

Randomization For all our devices, each assembled fabricated device was inspected for damage to the fiber optics, uPADs, or material. Once it passed inspection, the devices were randomly chosen for experiments with the desired wFDCF reaction.

Blinding Blinding is not relevant to our study. The data presented are quantitative values (see methods for how values were obtained) and did not require subjective judgment or interpretation. Blinding is not typically used in the field.

\section{Reporting for specific materials, systems and methods}

We require information from authors about some types of materials, experimental systems and methods used in many studies. Here, indicate whether each material, system or method listed is relevant to your study. If you are not sure if a list item applies to your research, read the appropriate section before selecting a response.

\begin{tabular}{|c|c|c|c|}
\hline \multicolumn{2}{|r|}{ Materials \& experimental systems } & \multicolumn{2}{|c|}{ Methods } \\
\hline $\mathrm{n} / \mathrm{a}$ & Involved in the study & $\mathrm{n} / \mathrm{a}$ & Involved in the study \\
\hline$\bigotimes$ & Antibodies & Х & $\square$ ChIP-seq \\
\hline Х & $\square$ Eukaryotic cell lines & $\bigotimes$ & $\square$ Flow cytometry \\
\hline Х & $\square$ Palaeontology and archaeology & $\bigotimes$ & $\square$ MRI-based neuroimaging \\
\hline Х & $\square$ Animals and other organisms & & \\
\hline Х & $\square$ Human research participants & & \\
\hline Х & $\square$ Clinical data & & \\
\hline Х & $\square$ Dual use research of concern & & \\
\hline
\end{tabular}

